\title{
RDC complex executes a dynamic piRNA program during Drosophila spermatogenesis to safeguard male fertility
}

\author{
Peiwei Chen $\mathbb{D}^{*}$, Yicheng Luo $\mathbb{1}$, Alexei A. Aravin $\mathbb{1}^{*}$ \\ California Institute of Technology, Division of Biology and Biological Engineering, Pasadena, California, \\ United States of America \\ * peiweitc@gmail.com (PC); aaa@ caltech.edu (AAA)
}

\section{G openaccess}

Citation: Chen P, Luo Y, Aravin AA (2021) RDC complex executes a dynamic piRNA program during Drosophila spermatogenesis to safeguard male fertility. PLoS Genet 17(9): e1009591. https:// doi.org/10.1371/journal.pgen.1009591

Editor: Jean-René Huynh, College de France CNRS, FRANCE

Received: November 1, 2020

Accepted: May 10, 2021

Published: September 2, 2021

Peer Review History: PLOS recognizes the benefits of transparency in the peer review process; therefore, we enable the publication of all of the content of peer review and author responses alongside final, published articles. The editorial history of this article is available here: https://doi.org/10.1371/journal.pgen.1009591

Copyright: @ 2021 Chen et al. This is an open access article distributed under the terms of the Creative Commons Attribution License, which permits unrestricted use, distribution, and reproduction in any medium, provided the original author and source are credited.

Data Availability Statement: All data are available within the manuscript except for the sequencing data, which is available at NCBI SRA (accession number: PRJNA646006).

\section{Abstract}

piRNAs are small non-coding RNAs that guide the silencing of transposons and other targets in animal gonads. In Drosophila female germline, many piRNA source loci dubbed "piRNA clusters" lack hallmarks of active genes and exploit an alternative path for transcription, which relies on the Rhino-Deadlock-Cutoff (RDC) complex. RDC was thought to be absent in testis, so it remains to date unknown how piRNA cluster transcription is regulated in the male germline. We found that components of RDC complex are expressed in male germ cells during early spermatogenesis, from germline stem cells (GSCs) to early spermatocytes. RDC is essential for expression of dual-strand piRNA clusters and transposon silencing in testis; however, it is dispensable for expression of Y-linked Suppressor of Stellate piRNAs and therefore Stellate silencing. Despite intact Stellate repression, males lacking RDC exhibited compromised fertility accompanied by germline DNA damage and GSC loss. Thus, piRNA-guided repression is essential for normal spermatogenesis beyond Stellate silencing. While RDC associates with multiple piRNA clusters in GSCs and early spermatogonia, its localization changes in later stages as RDC concentrates on a single $\mathrm{X}$ linked locus, $A T$-chX. Dynamic RDC localization is paralleled by changes in piRNA cluster expression, indicating that RDC executes a fluid piRNA program during different stages of spermatogenesis. These results disprove the common belief that RDC is dispensable for piRNA biogenesis in testis and uncover the unexpected, sexually dimorphic and dynamic behavior of a core piRNA pathway machinery.

\section{Author summary}

Large fractions of eukaryotic genomes are occupied by mobile genetic elements called transposons. Active transposons can move in the genome causing DNA damage and mutations, while inactive copies can contribute to chromosome organization and regulation of gene expression. Host cells employ several mechanisms to discriminate transposons from other genes and repress transposon activities. In germ cells, a conserved class of short RNAs called Piwi-interacting (pi)RNAs recognize target RNAs in both the nucleus and cytoplasm and then guide transposon repression by preventing their transcription 
Funding: This work was supported by the Howard Hughes Medical Institute Faculty Scholar Award (https://www.hhmi.org) and the National Institutes of Health R01 GM097363 (https://www.nih.gov) to AAA. The funders had no role in study design, data collection and analysis, decision to publish, or preparation of the manuscript.

Competing interests: The authors have declared that no competing interests exist. and destroying their RNAs. piRNAs are encoded in extended genomic regions dubbed piRNA clusters. Previously, composition and regulation of piRNA clusters were studied in the female germline of fruit flies, where a nuclear protein complex, the RDC complex, was shown to promote non-canonical transcription of these regions. However, RDC was believed to be dispensable in males. Here, we showed that RDC is essential for transposon repression in males, and males lacking RDC exhibit compromised fertility and loss of germ cells. We found that RDC binds multiple piRNA clusters in early germ cells but concentrates on a single locus at later stages. Our results indicate dynamic regulation of loci that produce piRNAs and, therefore, piRNA targets throughout spermatogenesis.

\section{Introduction}

Transposable elements (TEs) are selfish genetic elements that have the ability to propagate in the genome. When unchecked, transposition of TEs can cause overwhelming DNA damage and, eventually, genome instability. This poses a particular threat to germ cells, and TE derepression often leads to reproductive defects like sterility. To cope with this, a small RNAmediated genome defense mechanism involving PIWI proteins and PIWI-interacting RNAs (piRNAs) is employed in animal gonads to silence TEs [1].

In ovaries of Drosophila melanogaster, most piRNAs are made from so-called dual-strand piRNA clusters, where both genomic strands are transcribed to give rise to piRNA precursors. Transcription of dual-strand piRNA clusters is unusual in a number of ways. First, there is no clear promoter signature for transcription initiation [2]. Second, splicing, termination and poly-adenylation of nascent transcripts are all suppressed [2-4]. Third, transcription occurs at the presence of $\mathrm{H} 3 \mathrm{~K} 9 \mathrm{me} 3$ [5], a histone modification generally seen as a repressive mark for gene expression. In fact, canonical transcription must be repressed by factors like Maelstrom to allow proper piRNA production from dual-strand piRNA clusters [6]. Prior work has shown that such non-canonical transcription and co-transcriptional processing of piRNA precursors depend on the RDC complex, composed of Rhino (Rhi), Deadlock (Del) and Cutoff (Cuff) proteins, that bind dual-strand clusters [2,4]. Rhi belongs to the HP1 family and binds H3K9me3 through its chromo-domain, anchoring the RDC complex onto dual-strand clusters $[2,4,7]$. Cuff is a homolog of the conserved cap-binding protein Rail that was reported to suppress both splicing [4] and transcriptional termination [3], in order to facilitate the production of long, unspliced piRNA precursors. Del, on the other hand, recruits a paralog of transcription initiation factor TFIIA-L, Moonshiner (Moon), to initiate transcription in hostile heterochromatin environment [8]. Together, the RDC complex conveys transcriptional competence to dual-strand piRNA clusters, and the majority of piRNA production collapses when one of its components is missing.

While piRNA pathway is known to be active in both male and female germline of Drosophila, expression and functions of Rhi, Del and Cuff were studied exclusively during oogenesis. Mutations of rhi, del and cuff were shown to cause female sterility, however, mutant males remained fertile $[9,10]$. In addition, rhi, del and cuff are predominantly expressed in ovaries, with low or no expression in testes and somatic tissues [11-13]. Similarly, Moon was also believed to be ovary-specific, given that a high expression level could only be found in ovaries [8]. Finally, the silencing of Stellate by abundant Suppressor of Stellate piRNAs was shown to be unperturbed in testes of rhi and cuff mutants, suggesting that the RDC complex is dispensable for piRNA biogenesis in males $[14,15]$. Collectively, these findings led to the notion that 
RDC complex is dispensable for piRNA pathway in the male germline, raising the question of how piRNA cluster expression is controlled during spermatogenesis.

Here, we describe a developmentally regulated assembly of RDC complex in testes. We found that low expression of RDC complex components can be attributed to the fact that only a small subset of cells at early stages of spermatogenesis express rhi, del and cuff. Loss of RDC complex in testes results in a collapse of piRNA production, TE de-repression, and, ultimately, compromised male fertility, supporting an indispensable role of RDC complex in spermatogenesis. Even though RDC complex is assembled and functional in both sexes, we found differential genome occupancies of RDC complex between two sexes, correlating with sexually dimorphic usage of genome-wide piRNA source loci. Finally, RDC complex appears to exhibit dynamic binding on different piRNA clusters during spermatogenesis, allowing different piRNAs source loci to be used at different stages of early sperm development.

\section{Results}

\section{Components of the RDC complex are required for normal male fertility}

Previous studies showed that, while $r h i$ is required for female fertility, it is dispensable for male fertility $[13,14]$. In agreement with this, we found that $r h i$ mutant males indeed produce progeny when crossed with wildtype females. However, careful examination of the male fertility by sperm exhaustion test [16] revealed significantly compromised fertility in rhi mutant males. Even though most $r h i$ mutant males were initially fertile, the percentage of fertile males dropped as they aged and stayed low from day 3 in comparison to heterozygous sibling controls (Fig 1A). To probe male fertility more quantitatively, we repeated the test and counted numbers of progeny for each male every day. We found that even young, 1-day-old rhi mutant males, which were fertile, produced fewer progeny than heterozygous sibling controls (Fig 1A). Also, rhi mutant males produced nearly no progeny after two-day sperm exhaustion, while heterozygous sibling controls continued to produce $\sim 100$ progeny on average throughout the sperm exhaustion process. To extend this observation to other components of the RDC complex, we repeated the sperm exhaustion tests for del and cuff mutant males. Both del and cuff mutants displayed a reduction in male fertility compared to their respective heterozygous sibling controls ( $\mathrm{n}=5, P \leq 0.001, \mathrm{~S} 1 \mathrm{Fig}$ ) These results demonstrate that male fertility is substantially compromised at the absence of rhi, del or cuff, suggesting an indispensable role of all three RDC complex components in maintaining normal male fertility.

\section{RDC complex is assembled in nuclei of germ cells from GSCs to early spermatocytes}

The dependency of normal male fertility on rhi, del and cuff prompted us to re-examine whether RDC complex is assembled in testis. modENCODE data and previous work showed that tissue-wide mRNA levels of rhi, del and cuff are high in ovaries but low in testes and the soma [11-13], which led to the notion that RDC might be ovary-specific $[12,13,17]$. To examine expression of Rhi, Del and Cuff in testis, we took an imaging-based approach that provides single-cell resolution and preserves spatial information. We examined expression of individual components of the RDC complex using GFP-tagged Rhi, Del and Cuff transgenes that are expressed under the control of their native regulatory regions [2]. Importantly, GFP-tagged $\mathrm{RDC}$ components are functional, as their expression fully rescued the sterility of the respective female mutant (S2 Fig). All three proteins are expressed at the apical tip of testis that contains germ cells at early steps of spermatogenesis (Fig $1 \mathrm{~B}$ and $1 \mathrm{C}$ ), indicating that all three components of RDC complex are expressed in testis, though only in a small subset of the cells. 
A

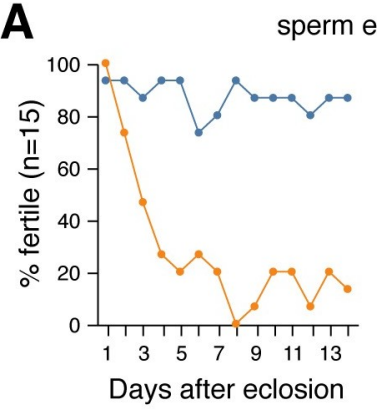

C

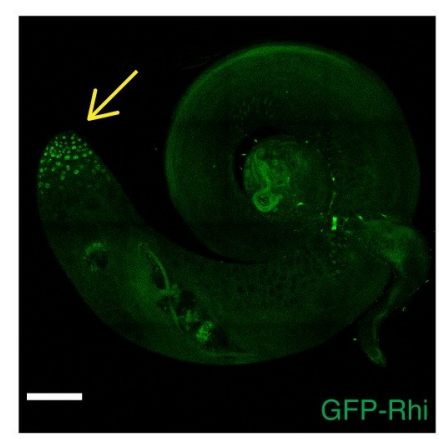

\section{xhaustion test}

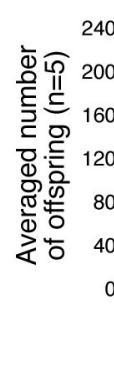

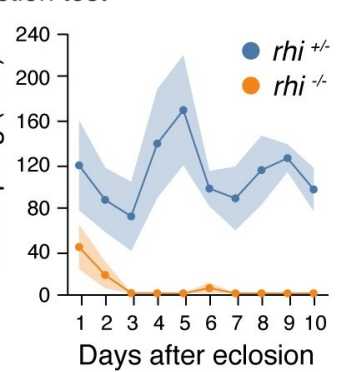

Days after eclosion
B

schematic of spermatogenesis

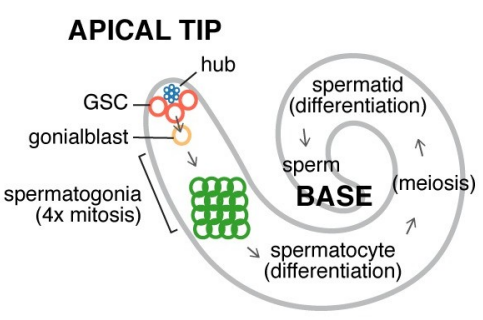

whole testis
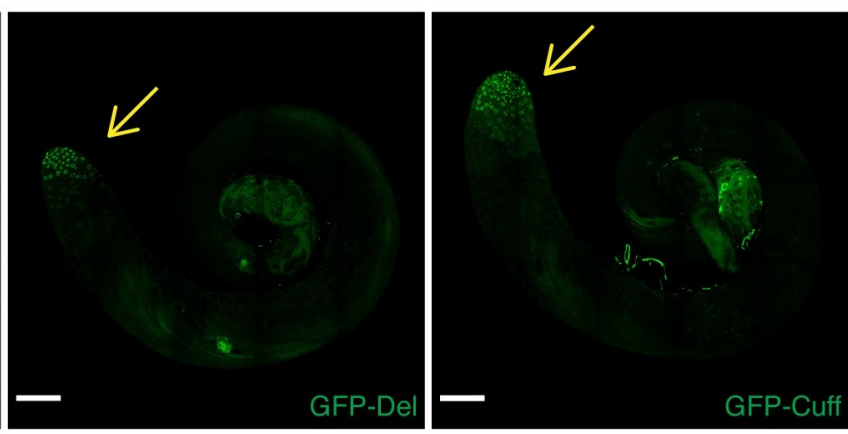

Fig 1. Rhi is required for normal male fertility, and components of RDC complex are expressed at the apical tip of testis. (A) Compromised fertility of rhi mutant males. Sperm exhaustion test of $r h i^{2 / K G}$ mutant (orange) and heterozygous sibling control (blue) males. Left: percentages of fertile males 1-14 days post-eclosion $(n=15)$. Right: averaged numbers of offspring per male 1-10 days after eclosion $(n=5)$. Shaded areas display standard error. Two charts report results from two independent sperm exhaustion tests. (B) A schematic of spermatogenesis, showing major developmental stages of male germline as well as the somatic hub that serves as germline stem cell (GSC) niche. (C) Expression of GFP-tagged Rhi (left), Del (middle) and Cuff (right) transgenes driven by their respective regulatory regions. Expression of all three proteins can only be seen at the apical tip of testis (pointed to by the yellow arrow). Scale bar: $100 \mu \mathrm{m}$.

\section{https://doi.org/10.1371/journal.pgen.1009591.g001}

Rhi, Del and Cuff form foci in nuclei (Fig 2A). To test whether all three proteins co-localize in nuclear foci, we tagged Rhi with a different fluorophore and expressed it using the previously described regulatory region of $r h i[4]$. After verifying that this transgene rescues female sterility of the rhi mutation (S2 Fig), we analyzed its localization with Del and Cuff. Indeed, Rhi co-localizes with both Del and Cuff (Fig 2A), consistent with the formation of RDC complex. Next, we examined the inter-dependence of Rhi, Del and Cuff localization (Fig 2B). In either del or cuff mutants, Rhi becomes dispersed and no longer forms puncta in nuclei. Similarly, Del also disperses at the absence of Rhi or Cuff. Expression of Cuff is strongly decreased in both $r h i$ and del mutants, indicating its destabilization. Therefore, Rhi, Del and Cuff colocalize in distinct nuclear foci that depend on the simultaneous presence of all three proteins.

We further characterized the expression of RDC complex in testis. Rhi, Del and Cuff are expressed in nuclei of germline stem cells (GSCs) that are directly adjacent to somatic hub cells labeled by Fas3, but not in hub cells (Fig 2C). Rhi expression continues beyond spermatogonia marked by Bam, until early spermatocytes that express Sa (ㅁpermatocyte arrest) (Figs $2 \mathrm{D}$ and $\mathrm{S} 3$ ). In bam mutant testes, where spermatogenesis is arrested at the spermatogonia-tospermatocyte transition stage, we observed an expansion of spermatogonia and expression of Rhi throughout entire testes (Fig 2E). In addition to germ cells and hub cells, the apical tip of testes contains somatic gonadal cells (cyst stem cells and early cyst cells) that can be distinguished from germ cells by $\mathrm{Tj}$ expression. Rhi is not expressed in somatic cells that express $\mathrm{Tj}$ 


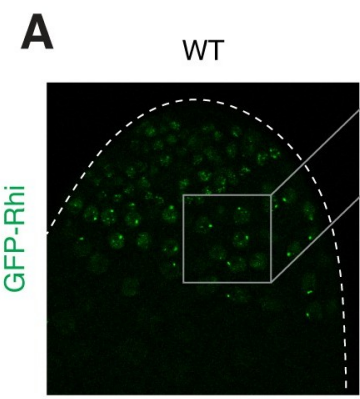

co-localization
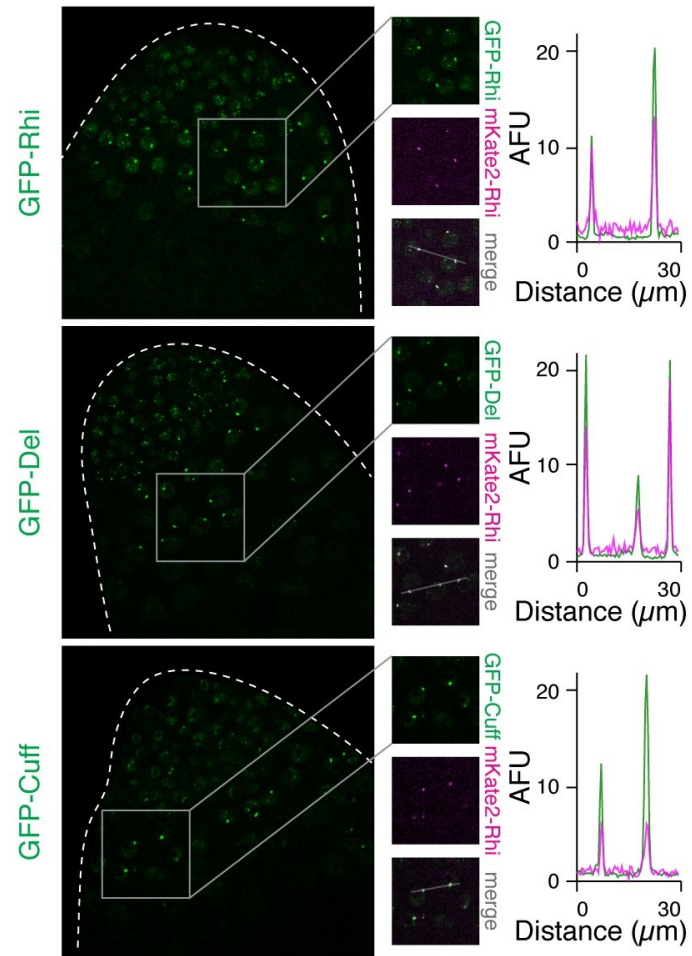

Distance $(\mu \mathrm{m})$
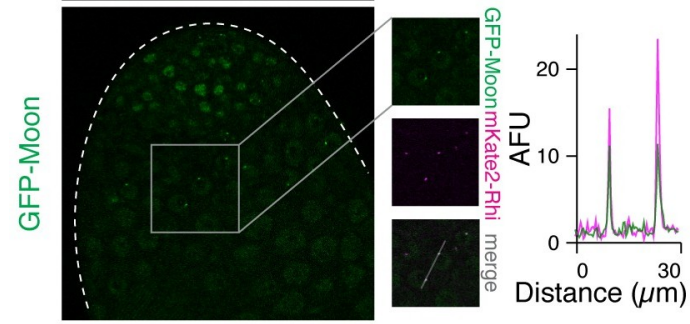

Distance $(\mu \mathrm{m})$

$\mathbf{D}_{\mathrm{t}}$ tj (early somatic gonadal cells)

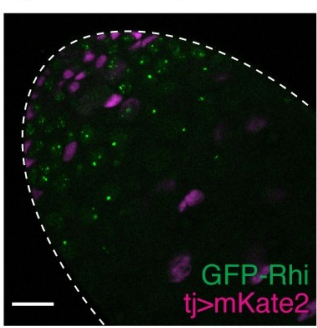

B
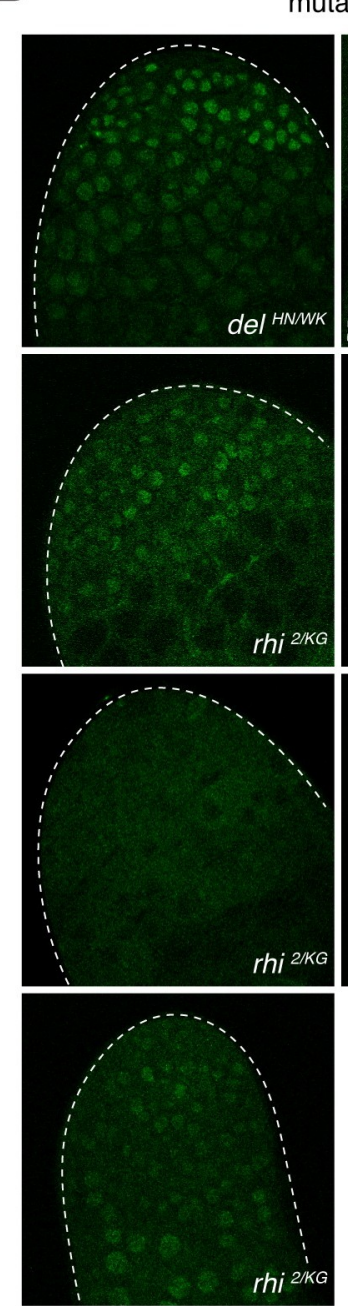

del HNWK
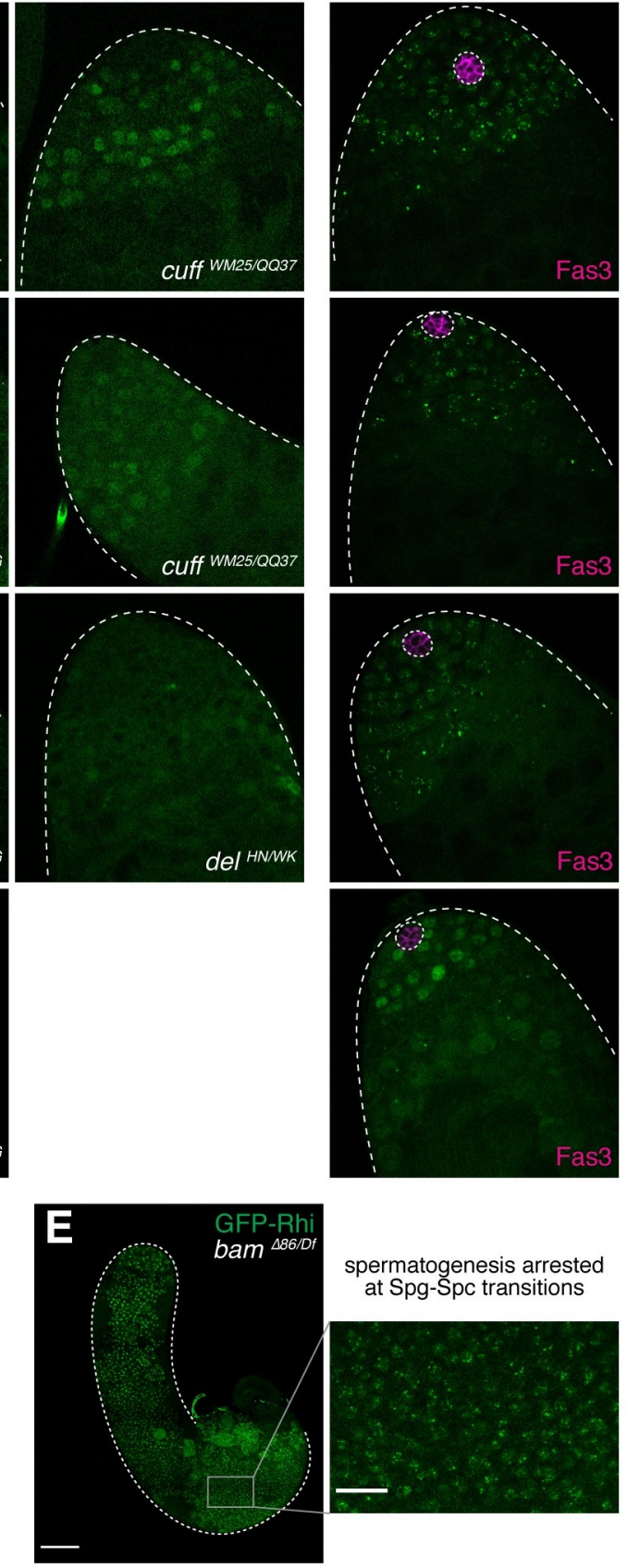

Fig 2. RDC complex is assembled in early male germ cells. (A) Rhi, Del, Cuff and Moon co-localize in nuclear foci. Confocal images showing apical tips of testes expressing GFP-tagged Rhi, Del, Cuff and Moon (top to bottom) transgenes driven by their native regulatory regions. Co-localization with mKate2-tagged Rhi in nuclear foci are shown on the right. Signal intensities along the marked line are plotted for each of four co-localization analysis. AFU, arbitrary fluorescence units. (B) Inter-dependence of Rhi, Del and Cuff localization in nuclear foci, as well as the dependence of Moon localization on Rhi. Confocal images showing apical tips of testes expressing GFP-Rhi in $\mathrm{del}^{\mathrm{HN} / W K}$ and $c u f f^{W M 25 / \mathrm{QQ} 37}$, GFP-Del in $r h i^{2 / K G}$ and $c u f f^{W M 25 / \mathrm{QQ} 37}$, GFP-Cuff in $r h i^{2 /}$ ${ }_{K G}$ and $d e l^{H N / W K}$, and GFP-Moon in $r h i^{2 / K G}$ mutant backgrounds. Nuclear foci of each protein dispersed or disappeared in respective mutants. (C) Rhi, Del, Cuff and Moon are expressed in GSCs. Immuno-fluorescence of testes expressing GFP-tagged Rhi, Del, Cuff and Moon, stained for somatic hub marker Fas3. Note that GSCs directly adjacent to Fas3-positive hub express all four proteins. (D) Rhi is not expressed in somatic gonadal cells, and its germline expression continues beyond spermatogonia till early spermatocytes. Confocal images showing a fluorescently tagged Rhi transgene with somatic gonadal cells marked by tj-Gal4>UASp-mKate2 (left), 4-16 cell spermatogonia marked by Bam-GFP (middle) and spermatocytes marked by Sa-GFP (right). Expression of Rhi in early spermatocytes is pointed to by yellow arrows. (E) Rhi expression upon arrest of spermatogenesis in bam mutants. Confocal image showing $b_{a m}^{\Delta 86 / D f}$ mutant testis expressing GFP-Rhi, where spermatogenesis is arrested at the spermatogonia-to-spermatocyte transition stage. Note 
that spermatogonia are expanded and virtually all germ cells express Rhi. An enlarged view of the basal part of mutant testis is shown at the bottom, which

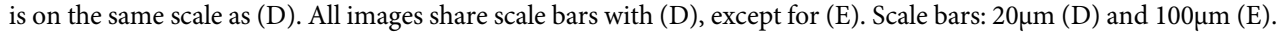

https://doi.org/10.1371/journal.pgen.1009591.g002

(Figs 2D and S3), confirming its restriction to the germline. Taken together, we conclude that RDC complex is assembled in male germ cells during spermatogenesis, from GSCs to spermatogonia and early spermatocytes.

In ovaries, RDC complex is known to promote piRNA cluster transcription by two mechanisms: 1) suppression of premature transcriptional termination, a function mediated by Cuff [3], and 2) licensing of non-canonical transcriptional initiation, a function that requires the recruitment of a basal transcriptional factor TFIIA-L paralog, Moonshiner (Moon) [8] . Expression of Moon was reported to be specific to female germline, raising the question of whether RDC complex can fulfill its function in the male germline, if its functional partner is missing. However, we observed Moon expression in testis using a GFP-tagged Moon transgene (expressed under its native regulatory region) that is able to rescue the female sterility caused by the moon mutation [8] (Fig 2A). Importantly, Moon is expressed at similar stages as components of RDC complex, from GSCs to spermatogonia and early spermatocytes at the apical tip of testis (Fig 2A and 2C). Furthermore, Moon co-localizes with Rhi in nuclear foci from late spermatogonia to early spermatocyte, and its focal localization is abolished in rhi mutants (Fig $2 \mathrm{~A}$ and $2 \mathrm{~B}$ ). In GSCs, Moon localization is more diffused than components of the RDC complex (Fig 2A and 2C). However, Moon expression is perturbed in rhi mutants, even in GSCs (Fig 2B), suggesting that Moon depends on Rhi for protein stability and proper localization in the nucleus throughout its expression window. On the contrary, Rhi localization appears normal in two different moon mutants, moon $^{\Delta 1}$ and moon $^{\Delta 28}$, indicating that Moon acts genetically downstream of RDC complex (S4 Fig). These observations suggest that RDC complex can recruit Moon to license transcription initiation in the male germline.

\section{Loss of RDC complex causes DNA damage and germ cell death in testis}

To identify cellular mechanisms underlying fertility decline in males lacking RDC complex, we examined the morphology of germ cells marked by Vasa-GFP in rhi mutants. Normally, Vasa-positive germ cells are tightly packed at the apical tip of testis, as we observed in testes of heterozygous control males $(\mathrm{n}=162$; Fig $3 \mathrm{~A})$. However, half of $r$ i mutant testes $(54.9 \%$, $\mathrm{n}=134 / 244$ ) had visibly fewer germ cells with prominent gaps in-between, indicative of an elevation of germ cell death (Fig $3 \mathrm{~A}$ ). Furthermore, another quarter of rhi mutant testes (25.4\%, $n=62 / 244)$ completely lost early germ cells altogether, and only $19.7 \%(n=48 / 244)$ showed wildtype-like germline morphology (Fig 3A and 3B). We concluded that loss of Rhi leads to a reduction in the germ cell count in testis.

Next, we examined impacts of Rhi loss on the resident germline stem cell (GSC) population. We quantified the number of GSCs per testis by counting the number of Vasa-positive germ cells directly adjacent in 3D to the somatic GSC niche labeled by Fas3. We found a reduction of GSCs in testes of rhi mutants compared with heterozygous controls in two age groups (1-4 and 9-12 days old) ( $P<0.0001$, Mann-Whitney-Wilcoxon test, Fig 3C). About a quarter of $r h i$ mutant testes did not have any GSC at all. Accordingly, we observed an expansion of Fas3-positive hub at a similar rate $(24.4 \%, \mathrm{n}=59 / 244)$, which usually occurs at the absence of GSCs and is never seen in control testes (Fig 3A). Hence, GSC population sizes shrink drastically in testes lacking Rhi. Similar to rhi mutants, testes of 10-day-old del and cuff mutants are often completely depleted of early germ cells including GSCs (Fig 3D), indicating that loss of any component of the RDC complex leads to a collapse of spermatogenesis. Notably, aged virgin mutant males that lost all early germ cells, nevertheless, harbor mature sperm in their seminal 
A

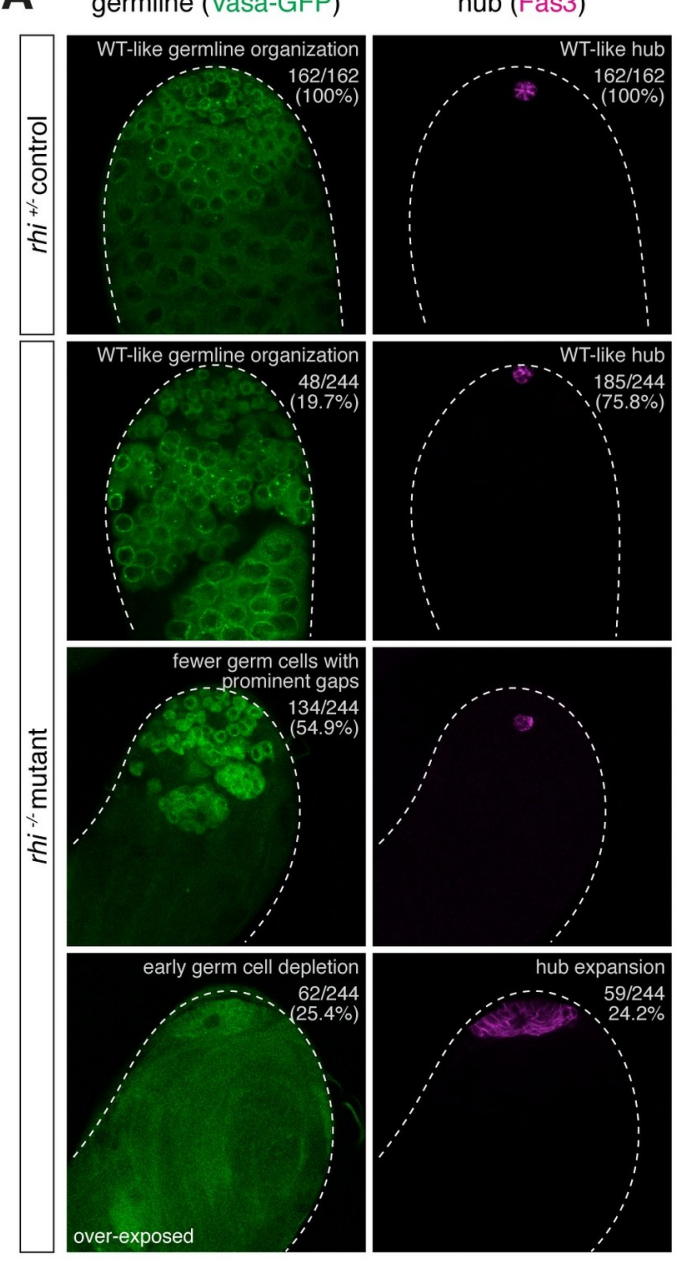

$\mathbf{F}$

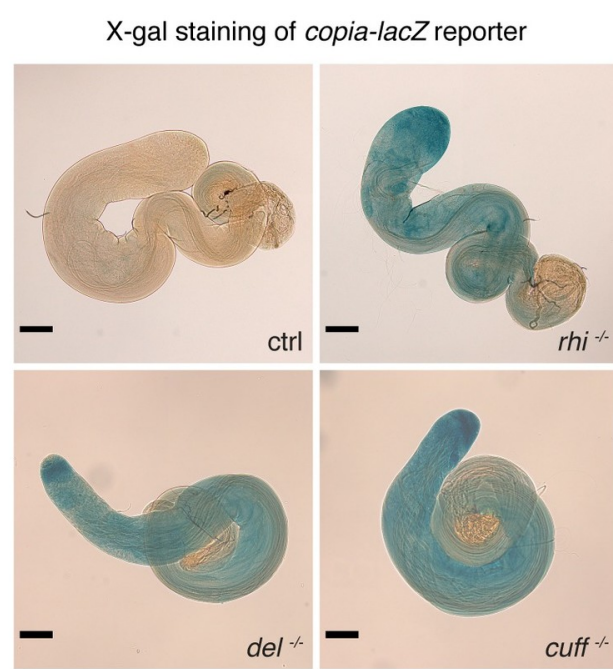

B

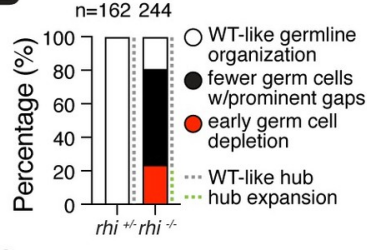

C

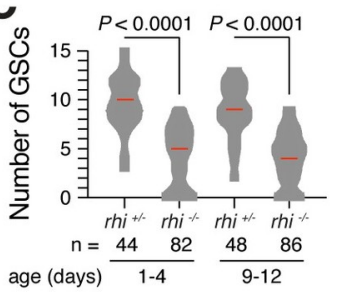

D of 10-day-old testis depleted of early germ cells del ${ }^{+/} 0 / 14 \quad$ del $\% 8 / 14$ cuff ${ }^{+/} 0 / 14$ cuff $\% 4 / 14$ example testis
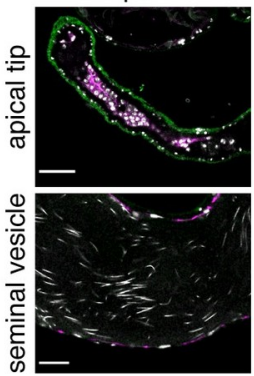

E

germline DNA double-strand breaks ( $\gamma-\mathrm{H} 2 \mathrm{Av}$ Vasa)
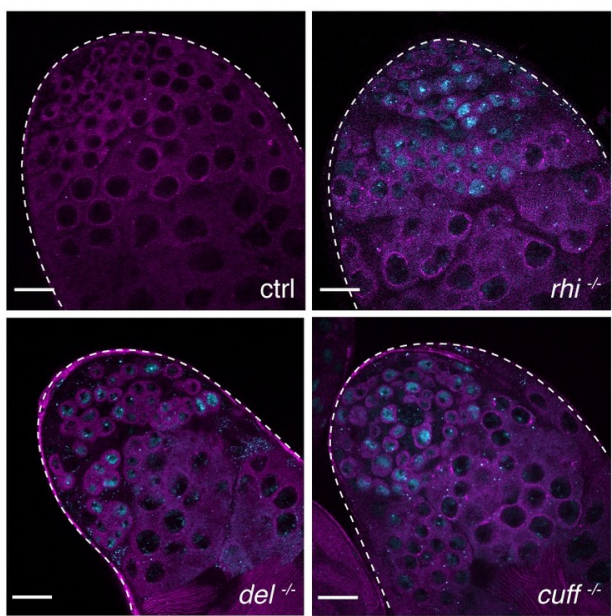

G

TE mRNAs

I

TE-AS piRNAs
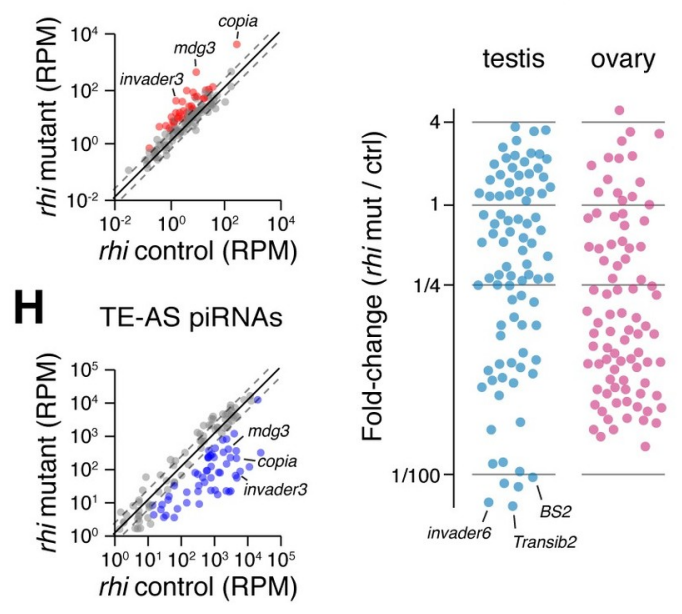

Fig 3. Loss of Rhi causes germ cell death, DNA damage and TE de-repression. (A) Loss of germ cells in testes of rhi mutants. Left: expression of germ cell marker, Vasa-GFP driven by vasa promoter, in testes of $r h i^{2 / K G}$ mutants and heterozygous control. Right: somatic hub cells that form a niche for GSCs are marked with Fas3. Classification of germ cell phenotype (defined by number and organization of germ cells that express Vasa) and hub size phenotype (defined by cells that express Fas3) are labeled at the top right with corresponding statistics. All images on the same scale as (D). (B) Quantification 
of germ cell survival and hub size in rhi heterozygous control (left) and mutant (right) testes shown in (A). n, number of testes examined. (C) Loss of GSCs in testes of $r h i$ mutants. Violin plot showing GSC numbers in $r h i^{2 / K G}$ mutant and age-matched heterozygous sibling control testes. Median of GSC number is marked red. GSC number is acquired by counting the total number of Vasa-positive cells directly adjacent to Fas3-positive hub in 3D. $P<0.0001$ based on Mann-Whitney-Wilcoxon test. n, number of testes counted for each genotype and age group. (D) Testes of del and cuff mutant males showed frequent loss of early germline but produce mature sperm indicating no block of spermatogenesis. Number of 10-day-old virgin males depleted of early germline is listed for each genotype at the top. The apical tip of testis and the seminal vesicle (SV) of such testes are shown below as an example, stained for Vasa, Fas3 and DAPI. Scale bar: $40 \mu \mathrm{m}$ and $20 \mu \mathrm{m}$ for testis and SV, respectively. (E) Accumulation of DNA double-strand breaks (DSBs) in male germline of $r h i^{2 / K G}$, $\mathrm{del}^{\mathrm{HN} / W K}$ and cuff ${ }^{W M 25 / \mathrm{QQ} 37}$ mutants. Immuno-fluorescence of heterozygous control and mutant testes, stained for $\gamma-\mathrm{H} 2 \mathrm{Av}$, a marker for DNA DSBs, and Vasa, a germline marker. Scale bars: $20 \mu \mathrm{m}$. (F) De-repression of copia reporter in testes of $r h i$ mutants. Brightfield images showing heterozygous control and $r h i^{2 / K G}$, del ${ }^{H N / W K}$ and cuff ${ }^{W M 25 / Q Q 37}$ mutant testes expressing copia-lacZ, after X-gal staining. copia LTR containing its promoter is fused upstream to lacZ gene. Note that part of copia LTR is transcribed as well. Scale bar: $100 \mu \mathrm{m}$. (G) De-repression of TEs in testes of $r h i$ mutants measured by polyA+ RNA-seq. Scatter plot showing expression of TE mRNAs in $r h i^{2 / K G}$ mutant versus heterozygous control testes. TEs that show $\geq 2$-fold de-repression $($ FDR $<0.05)$ and $\geq 1$ RPM averaged levels are marked red. The mean of two biological replicates is shown. (H) Loss of TEtargeting piRNAs in testes of $r h i$ mutants. Scatter plot showing expression of TE-antisense piRNAs in $r h i^{2 / K G}$ mutant versus heterozygous control testes. TE-antisense piRNAs that show $\geq 2$-fold reduction $(F D R<0.05)$ and $\geq 10$ RPM averaged levels are marked blue. Shown are averages of two biological replicates. (I) Loss of TE-targeting piRNAs in rhi males and females. Scatter plot showing fold-change of TE-antisense piRNAs upon loss of rhi in testis (left, blue) and ovary (right, pink). Note that piRNAs targeting several TE families demonstrate over 100-fold reduction in rhi testis, a magnitude not observed in ovary. Averages of two biological replicates are shown.

https://doi.org/10.1371/journal.pgen.1009591.g003

vesicles (Fig 3D), suggesting that disruption of the RDC complex does not block spermatogenesis at a specific stage in young males, but early germ cells are depleted when they age. Staining testes for the phosphorylated H2A variant $(\gamma-\mathrm{H} 2 \mathrm{Av})$, a marker for DNA double-strand breaks (DSBs), revealed massive accumulation of unrepaired DNA DSBs in early germ cells of $r h i, d e l$ and cuff mutant testes (Fig 3E). Unrepaired DNA DSBs likely cause germ cell death in mutants of RDC complex components. Overall, our results suggest that the loss of early germ cells, including GSCs, accompanied by widespread unrepaired DNA DSBs is responsible for the compromised fertility of mutant males lacking an intact RDC complex.

\section{RDC complex is required for TE silencing in testis}

Widespread DNA DSBs can result from TE transposition. To quantify TE expression, we sequenced polyadenylated (polyA+) RNAs from rhi mutant and control testes. PolyA+ RNAseq revealed $32 \mathrm{TE}$ families showing significant up-regulation in testes of $r$ r $i$ mutants $(>2$-fold increase, FDR $<0.05$; Fig 3G). Among them, the most de-repressed ones include $m d g 3$ (36-fold), invader3 (18-fold) and copia (13-fold). To verify TE de-repression, we employed a copia-lacZ reporter, where the long terminal repeat (LTR) of copia containing copia promoter is fused upstream to the lac $Z$ gene and its expression can be directly examined by $\mathrm{X}$-gal staining [18]. copia is known to be active in the male germline [19] and has the highest expression level among all TEs in testes [20]. Whereas no detectable X-gal staining was seen in control testes, robust staining was observed in rhi mutants (Fig 3F), confirming strong de-repression of copia at the absence of rhi. Similarly, the copia reporter was de-repressed in del and cuff mutant testes (Fig 3F). These results show that TEs are de-repressed in testes lacking a functional RDC complex.

In ovaries, RDC complex is required for piRNA production from dual-strand clusters to ensure efficient TE silencing [2-4,8]. To examine piRNA biogenesis, we sequenced and analyzed small RNAs in rhi mutant and control testes. We found a loss of antisense piRNAs targeting many TE families in $r h i$ mutant testes ( $>2$-fold reduction, FDR $<0.05$; Fig $3 \mathrm{H}$ ), suggesting an overall defect in piRNA production. In fact, there is a moderate correlation between the fold-derepression of TEs and the fold-reduction of TE-targeting piRNAs in testis upon mutating $r h i\left(\log\right.$-transformed values: Spearman's $\rho=0.41, P=7.3 \times 10^{-5}$, Pearson's $\rho=$ 
$\left.0.46, P=8.4 \times 10^{-6}\right)$. For TEs showing strong up-regulation in rhi mutant testes, we observed a concurrent, pronounced loss of antisense piRNAs (e.g., mdg3, invader 3 and copia). Notably, there are antisense piRNAs against several TE families (e.g., BS2, Transib2, invader6) that show over 100-fold reduction in rhi mutant testes, a magnitude not observed for any TE family in rhi mutant ovaries (Fig 3I). Finally, sense piRNAs were also lost for many TE families (S5 Fig), consistent with dual-strand clusters producing piRNAs from both genomic strands in a Rhi-dependent manner. These results show that efficient production of TE-silencing piRNAs in testis depends on the RDC complex, without which many TEs are de-repressed, causing DNA damage and germ cell death in testis.

\section{RDC complex is required for piRNA production from dual-strand clusters in early male germ cells}

To understand the role of RDC complex in piRNA cluster expression in testis, we analyzed effects of rhi mutation on piRNA production from major piRNA clusters. Genomic loci that generate piRNAs in testis were recently de novo defined leading to identification of several novel piRNA clusters [20]. Rhi was dispensable for expression of uni-strand piRNA clusters, flam and 20A (Fig 4A), similar to results from ovary studies [2,4,14]. Surprisingly, we found that Rhi was also dispensable for piRNA production from the Y-linked $\mathrm{Su}(\mathrm{Ste})$ locus, which is the most active piRNA cluster in testis [20]. Unlike flam and 20A, Su(Ste) is a dual-strand cluster that generates piRNAs from both genomic strands. We confirmed by RNA fluorescence in situ hybridization (FISH) that piRNA precursor transcription from $\mathrm{Su}(\mathrm{Ste}$ ) appeared intact in testes without Rhi (Fig 4C). In contrast to $\mathrm{Su}(\mathrm{Ste}$ ), piRNA production from other major dualstrand clusters, including the Y-linked petrel cluster, collapses in rhi mutant testes (Fig 4A), indicating that expression of the majority of dual-strand piRNA clusters in testis relies on Rhi. Interestingly, dependence of dual-strand cluster expression on Rhi varies between sexes: $38 \mathrm{C}$ is more affected by loss of $r h i$ than $42 A B$ in testis, while the opposite is found in ovary. Furthermore, piRNA production from both strands of complex satellites, which we recently found to behave as dual-strand piRNA clusters [20], also drastically declined in rhi mutant testes and ovaries (Fig 4B). These results show that Rhi is essential for piRNA production from a large fraction of piRNA clusters in the male germline.

Since RDC complex forms distinct foci in the nuclei of germ cells (Fig 2A), we set out to test if Rhi binds the chromatin of dual-strand clusters whose expression depends on RDC complex, as reported in ovary $[2,4,14]$. Given that expression of RDC complex is restricted to a small number of cells at the apical tip of testis, we used bam mutant testes, where Rhi-expressing spermatogonia are expanded (Fig 2E), to perform ChIP-seq of Rhi. All major dual-strand clusters, with the exception of $S u$ (Ste), were enriched for Rhi binding (Fig 4D). In agreement with ChIP-seq, independent ChIP-qPCR showed no evidence of Rhi binding on $\mathrm{Su}(\mathrm{Ste})$ locus $(\mathrm{n}=4)($ Fig $4 \mathrm{G})$. Rhi was also absent on chromatin of uni-strand clusters, flam and 20A. Importantly, the binding of Rhi on different loci seems to correlate with its effect on promoting piRNA cluster expression. Rhi does not bind, and is dispensable for piRNA production from, uni-strand clusters and $S u(S t e)$, while it binds, and is required for expression of, other dual-strand clusters (Fig 4A and 4D). Also, dual-strand clusters that show the highest levels of overall Rhi binding, 38C and AT-chX, demonstrate the strongest Rhi dependence for piRNA production. To characterize the relationship between Rhi binding and piRNA production on a genome-wide scale, we analyzed Rhi binding and piRNA production in $1 \mathrm{~Kb}$ genomic windows spanning the entire genome (Fig 4E). For loci that depend on Rhi to produce piRNAs, we observed a strong correlation between Rhi binding and piRNA levels (Spearman's $\rho=0.97$; Pearson's $\rho=0.99$ ). On the other hand, loci that continue to produce piRNAs at the absence of 
A
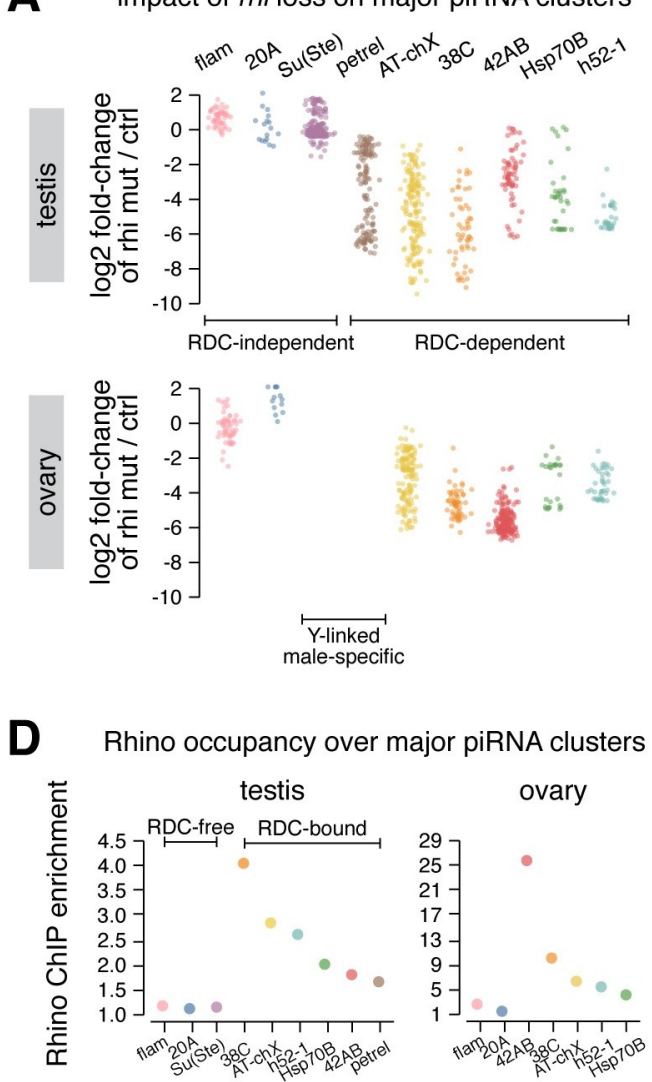

B impact of $r h i$ loss on satellite piRNAs

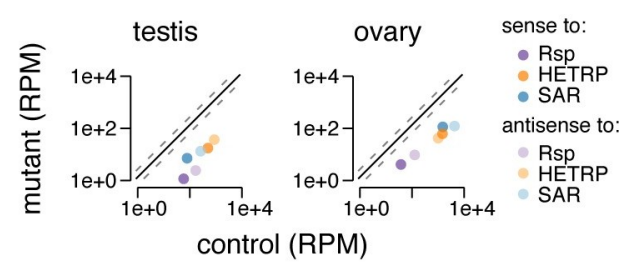

C RNA FISH of Su(Ste) piRNA precursors

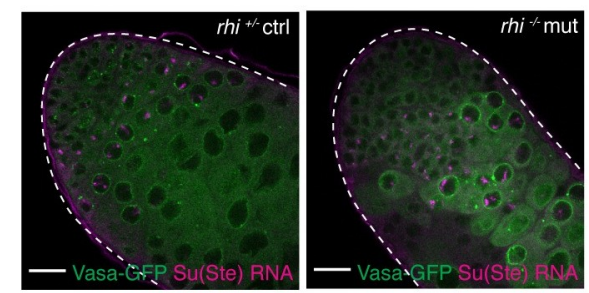

E

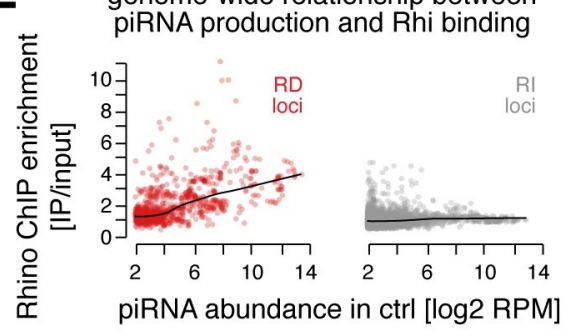

$\mathbf{F}$

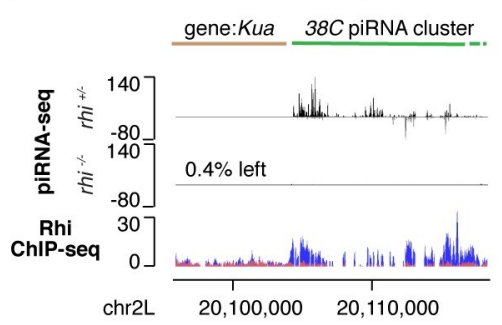

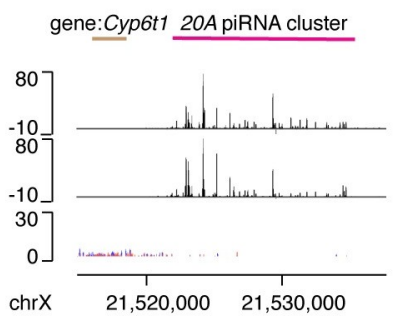

G Rhi ChIP-qPCR $(\mathrm{n}=4)$

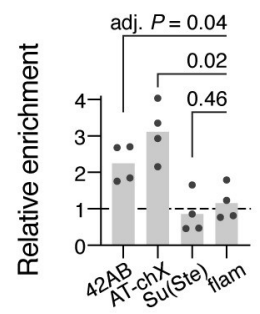

Fig 4. RDC complex is required for piRNA production from dual-strand piRNA clusters. (A) Impacts of rhi loss on piRNA production from major piRNA clusters in testis and ovary. Scatter plot showing fold-change of piRNA production from $1 \mathrm{~Kb}$ genomic windows spanning major piRNA clusters in testis (top) and ovary (bottom), upon loss of rhi. Two Ylinked, male-specific clusters are not present in female genome. Each cluster is given a unique color. Averages of two biological replicates are shown. (B) Impact of $r h i$ loss on piRNA production from satellite repeats in testis and ovary. Scatter plot showing levels of complex satellite-mapping piRNAs in $r h i^{2 / K G}$ mutant and control testis (left) and ovary (right). Each complex satellite is assigned a color, and piRNAs sense to satellite consensus are marked with higher opacity than antisense ones. Mean of two biological replicates is shown. (C) Unperturbed expression of $\mathrm{Su}(\mathrm{Ste})$ piRNA precursors in testes of rhi mutants. RNA fluorescence in situ hybridization of $S u(S t e)$ piRNA precursors in $r h i$ control (left) and $r h i^{2 / K G}$ mutant (right) testes that express Vasa-GFP. Scale bar: $20 \mu \mathrm{m}$. (D) Rhi occupancy over major piRNA clusters in testis and ovary. Scatter plot showing Rhi ChIP-seq enrichment over major piRNA clusters in testis (left) and ovary (right). Shown are averages of two biological replicates. Two Y-linked, male-specific clusters are not present in female genome. Each cluster is colored the same way as in (A). (E) Scatter plot showing the relationship between Rhi ChIP enrichment and piRNA production over $1 \mathrm{~Kb}$ genomic windows. Loci that show $\geq 4$-fold decline in piRNA production at the absence of $r h i$ are defined as Rhi-dependent ("RD", red), otherwise Rhi-independent ("RI", gray). Each dot is the average of two biological replicates. Black lines show local regression. (F) Examples of Rhi-dependent (38C, left) and Rhi-independent (20A, right) piRNA clusters. piRNA-seq in rhi mutant and control testes are shown at the top, and Rhi ChIP-seq is shown at the bottom (blue: IP, red: input). The profile of a representative replicate is shown. (G) Rhi does not bind $\mathrm{Su}(\mathrm{Ste})$ cluster in testis. Bar graphs showing Rhi ChIP-qPCR $(\mathrm{n}=4)$ over four piRNA clusters in testis. Adjusted $P$-values are from multiple t-tests corrected for multiple comparisons by the Holm-Sidak method. Uni-strand piRNA cluster flam not bound by Rhi serves as a negative control.

https://doi.org/10.1371/journal.pgen.1009591.g004 
Rhi usually have little, if any, Rhi binding. Collectively, our data indicate that Rhi physically binds the chromatin and ensures the expression of dual-strand piRNA clusters, with a notable exception of $S u(S t e)$.

\section{Sexually dimorphic genome occupancy of RDC complex sculpts sex- specific piRNA program}

piRNA profiles are distinct in male and female gonads, and expression of dual-strand piRNA clusters are sexually dimorphic [20]. To explore if RDC complex might be involved in orchestrating sex-specific piRNA programs, we profiled Rhi binding on the genome in ovaries under identical ChIP-seq conditions as in testes. This analysis revealed differences in Rhi genome occupancy between sexes among top piRNA clusters (Fig 5A). For example, Rhi is more enriched on $38 \mathrm{C}$ than $42 \mathrm{AB}$ in testes, whereas the reciprocal is seen in ovaries, correlating with differential piRNA production from these two loci in two sexes. In addition, $80 E F$ and $40 F 7$ clusters have high levels of Rhi binding in ovary but low in testis, mirrored by abundant piRNA production from these two loci in ovary but not in testis. Finally, an ovary-specific dual-strand piRNA cluster, Sox102F, is bound by Rhi in ovary, while there is no evidence of Rhi binding at Sox $102 F$ in testis where it is inactive (Fig $5 \mathrm{~B}$ ). Altogether, the observed link between Rhi binding and piRNA production between males and females suggests that the sex-specific Rhi binding on piRNA clusters is responsible for sculpting a sexually dimorphic piRNA program.

\section{RDC complex enables dynamic piRNA production during spermatogenesis}

ChIP-seq provides the genome-wide profile of Rhi binding, but it masks possible differences of Rhi localization among individual cells. Imaging of Rhi revealed distinct Rhi localization in nuclei of germ cells at different stages of spermatogenesis (Fig 6A). In the nuclei of GSCs and
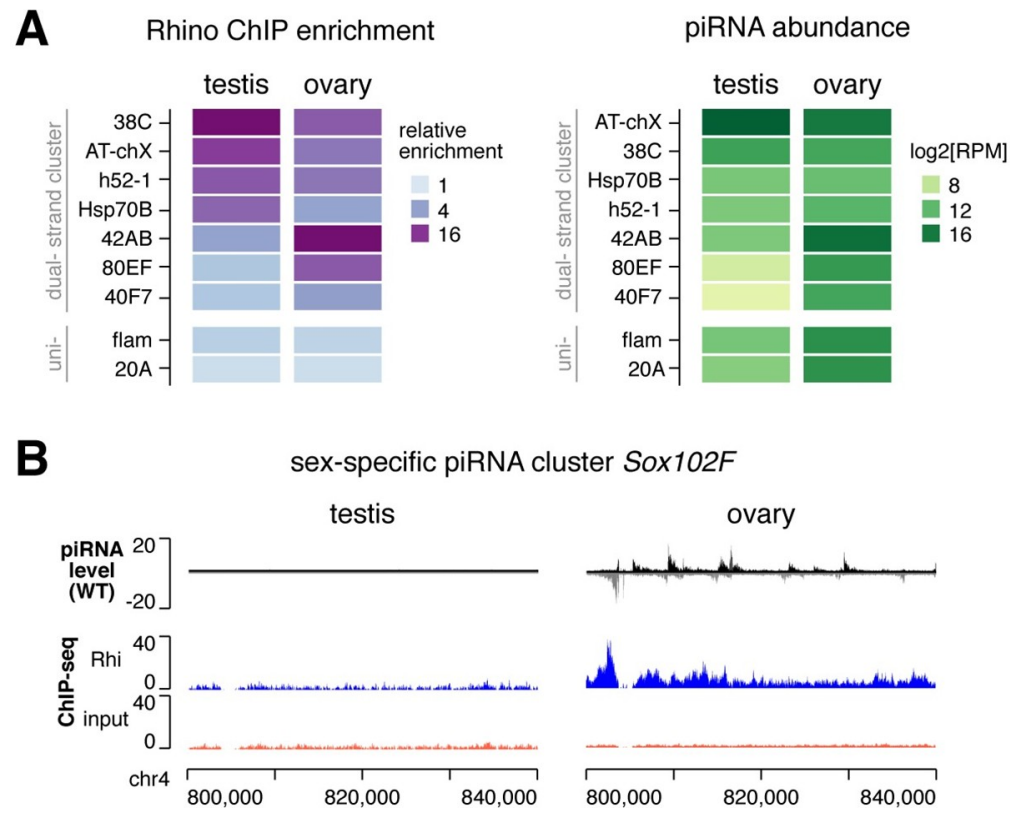

Fig 5. Sexually dimorphic RDC genome occupancy sculpts sex-specific piRNA program. (A) Heatmaps showing relative enrichment of Rhi binding over major piRNA clusters determined by ChIP-seq (left) and piRNA production from major piRNA clusters (right) in two sexes. The mean of two biological replicates is shown. Rhi-dependent dualstrand clusters are shown at the top, and Rhi-independent uni-strand clusters at the bottom. (B) Sox102F piRNA cluster produces piRNAs exclusively in ovary. Rhi is enriched on Sox $102 \mathrm{~F}$ in ovary, but not in testis. Shown is the profile from a representative replicate.

https://doi.org/10.1371/journal.pgen.1009591.g005 
A

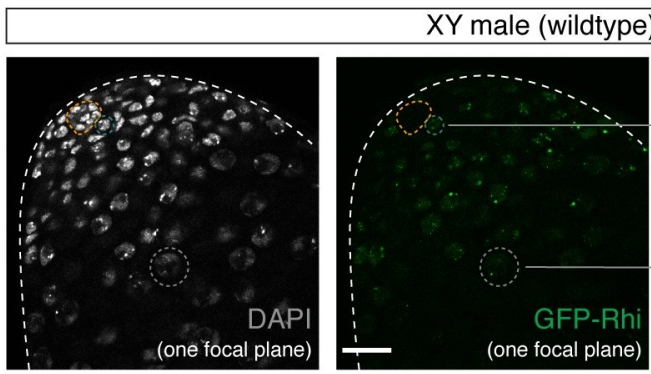

C

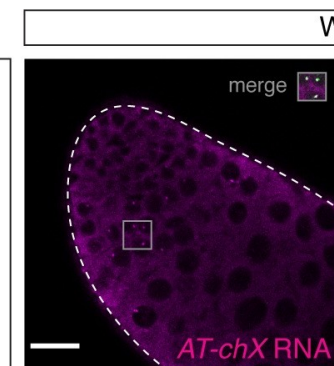

WT
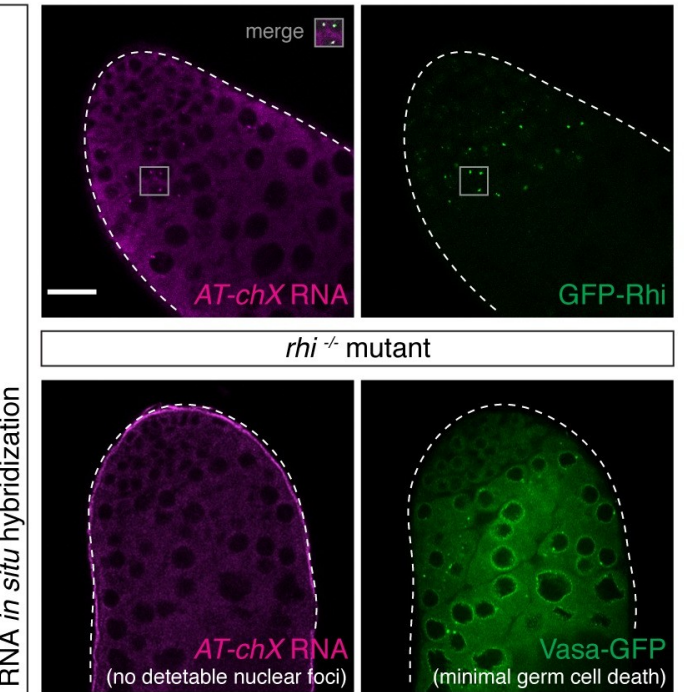

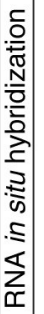
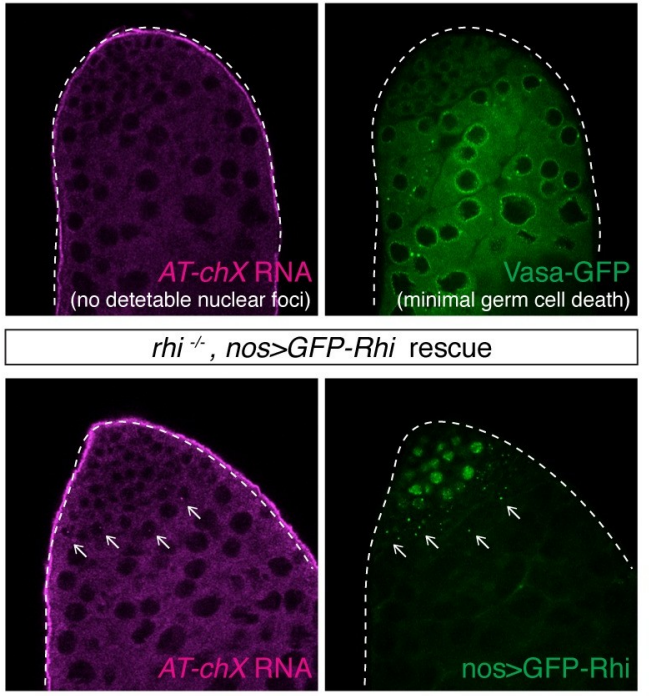

H

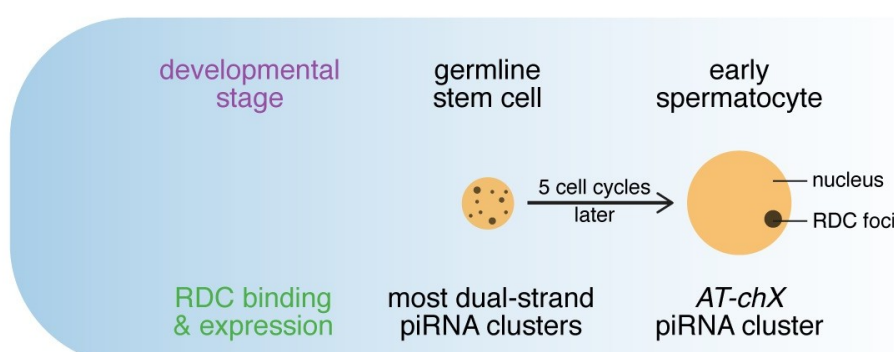

B

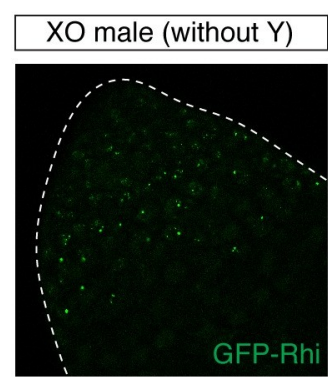

D

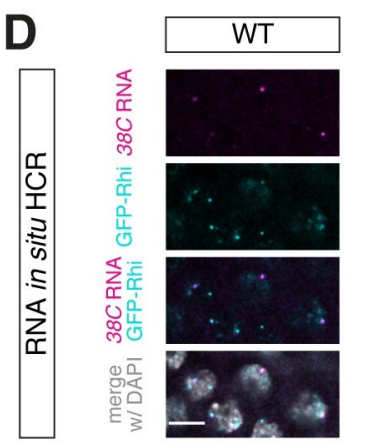

E

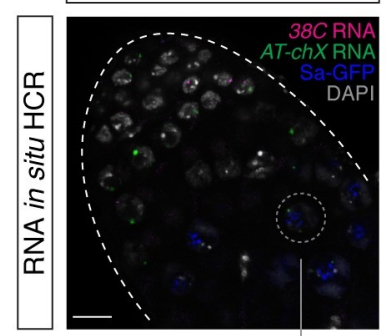

$87.7 \%(n=50 / 57)$ early spermatocytes that express AT-chX do not express $38 \mathrm{C}$ anymore

occupies many loci across the genome

early spermatocyte: concentrates onto one single locus

(max intensity projection)
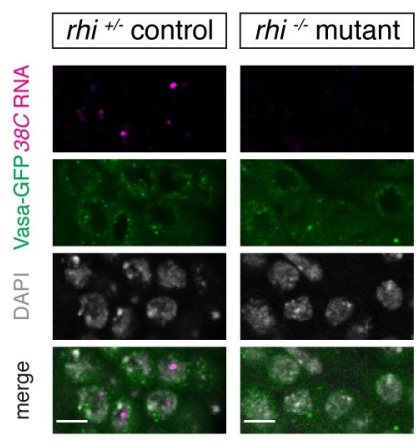

$\mathbf{F}$

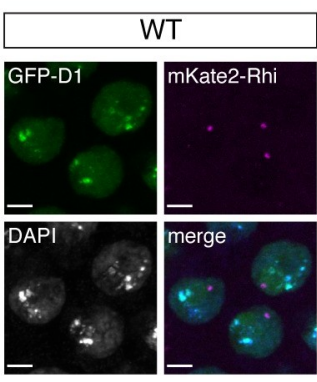

(max intensity projection)

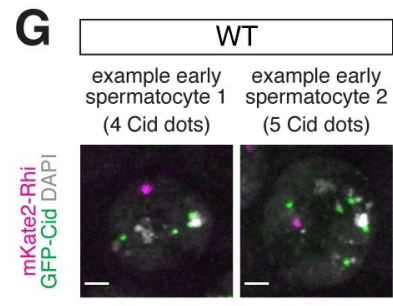

(max intensity projection)

Fig 6. RDC complex enables dynamic piRNA production during early spermatogenesis. (A) Rhi localizes to multiple nuclear foci in GSCs and early spermatogonia but concentrates in a single dot in early spermatocytes. Confocal images of the apical tip of testis expressing GFP-Rhi transgene. A single focal plane is shown for DAPI and GFP-Rhi, while maximum intensity projections covering entire nuclei are shown for a GSC and an early spermatocyte on the right. Orange circle outlines the somatic hub for identification of GSCs next to it. Early spermatocyte is identified by formation of chromosome territory and increased nuclear size by DAPI. (B) Rhi localization to a single nuclear dot is not affected in early spermatocytes that lack Y chromosome. Confocal image of the apical tip of testis from XO male (that lacks Y chromosome) expressing 
GFP-Rhi transgene. (C) Rhi localizes exclusively to $A T$-chX piRNA cluster in early spermatocytes. Top: RNA fluorescence in situ hybridization (FISH) of $A T$-ch $X$ piRNA precursors in wildtype testis expressing GFP-Rhi. Middle: RNA FISH of $A T-\operatorname{ch} X$ piRNA precursors in $r h i^{2 / K G}$ mutant testis. Note that the absence of $A T$-chX RNA foci is not due to the loss of germ cell as Vasa expression shows minimal germ cell death in this particular testis. Bottom: expression of Rhi transgene driven by nos-Gal4 rescues expression of $A T$-chX cluster in testis of $r h i^{2 / K G}$ mutant. (D) Rhi binds 38C piRNA cluster in spermatogonia. RNA in situ hybridization chain reaction (HCR) of 38C piRNA precursors in testis expressing GFP-Rhi (left) or Vasa-GFP in heterozygous control and rhi mutant background (right). Shown are spermatogonia as indicated by DAPI staining. Note that, in contrast to exclusive co-localization with $A T$-chX cluster in spermatocytes shown in (C), only a subset of Rhi foci co-localize with 38C RNA foci, indicating that Rhi binds other piRNA clusters besides 38C in spermatogonia. 38C RNA signal is present in control but absent in rhi mutant testes. (E) $A T-\operatorname{chX}$ and $38 C$ co-express in spermatogonia, but only $A T$-chX is expressed in most early spermatocytes. Dual in situ HCR of $38 C$ and $A T$-chX piRNA precursors in testis expressing Sa-GFP (marker for spermatocytes). Circled is an example of early spermatocytes that express $A T-c h X$ but not $38 \mathrm{C}$. Quantification is shown at the bottom. Note that co-expression of $38 C$ and $A T$-chX can be seen in Sa-negative spermatogonia. (F) D1 and Rhi do not co-localize in early spermatocytes. Confocal image of early spermatocytes expressing GFP-D1 (a protein trap line) and mKate2-Rhi driven by the rhi promoter. (G) Rhi is localized in a single dot in spermatocyte nuclei, where individual chromosomes occupy distinct chromosomal territories. Confocal image of early spermatocytes expressing mKate2-Rhi and the centromere marker GFP-Cid. The presence of four or more Cid foci indicates that centromeres of individual non-homologous chromosomes are not clustered together. (H) Proposed model of how Rhi switches genomic binding sites during gametogenesis from GSC to early spermatocyte stage, in order to allow dynamic employment of different piRNA clusters. Scale bars: $20 \mu \mathrm{m}$ (A-C, E), $5 \mu \mathrm{m}(\mathrm{D}), 4 \mu \mathrm{m}(\mathrm{F})$ and $2 \mu \mathrm{m}(\mathrm{G})$.

https://doi.org/10.1371/journal.pgen.1009591.g006

spermatogonia, Rhi forms many discrete foci, suggesting-in agreement with ChIP-seq resultsthat it binds multiple genomic loci. As male germ cells differentiate into spermatocytes and prepare for meiosis, however, Rhi concentrates as one single dot in nuclei of early spermatocytes, suggesting its specific localization at one single locus. Notably, while homologous chromosomes pair in about half of late spermatogonia and very early spermatocytes [21], we only observed one bright Rhi dot in virtually all nuclei at these stages, suggesting that this locus resides on one of the two sex chromosomes rather than an autosome. To explore where Rhi binds at this stage, we first examined testes from $\mathrm{XO}$ males that lack the $\mathrm{Y}$ chromosome and found that Rhi still localizes as one single dot in early spermatocytes, arguing against Y-linked loci such as $\mathrm{Su}(\mathrm{Ste}$ ) and petrel (Fig 6B). Simultaneous imaging of Rhi and RNA FISH of transcripts from the X-linked $A T$-chX locus revealed co-localization of $A T$-chX nascent transcripts and Rhi in one single dot from late spermatogonia to early spermatocytes, indicating that Rhi concentrates on $A T$-chX locus at this stage (Fig 6C). Indeed, single $A T$-chX RNA foci in individual nuclei became undetectable in rhi mutant testes, and expressing GFP-tagged Rhi transgene by nanos-Gal4 in rhi mutant background restored $A T$-chX expression (Fig 6C). Importantly, even though nanos-Gal4 drives stronger expression of GFP-Rhi in earlier stages (GSC and early spermatogonia), $A T$-chX transcripts remained highly expressed specifically during later stages (late spermatogonia and early spermatocyte) (Fig 6C). This finding suggests that the spatio-temporally regulated gene expression of $A T$-chX piRNA cluster is rather robust to perturbations to the Rhi protein level, and the low expression of $A T$-chX piRNA cluster earlier in GSCs and early spermatogonia is not limited by the level of Rhi protein. In sum, these results show that Rhi binds multiple genomic loci in GSCs and spermatogonia but appears to concentrate on a single $A T$-chX locus later.

As Rhi is required for non-canonical transcription of dual-strand piRNA clusters, depletion of Rhi from clusters other than $A T-c h X$ should cease their expression. To test this, we set out to conduct RNA FISH of piRNA precursors from other Rhi-dependent dual-strand piRNA clusters (Fig 5A). Previously, FISH detection of piRNA precursor transcripts was performed in polyploid nurse cells in fly ovary, whose genome is endo-replicated up to $1032 \mathrm{C}$ with a much higher expression level of piRNA precursor transcripts [2,8,22]. In addition to low expression levels in the diploid male germline, piRNA cluster transcripts are difficult to target, as they are highly repetitive and share extensive sequence homology with TEs. To tackle these challenges, we employed in situ hybridization chain reaction (HCR), which permits enzyme-free signal amplification and automatic background suppression [23], to target transcripts from $38 \mathrm{C}$ 
piRNA cluster that has a relatively high expression level in testis (Fig 5A). in situ HCR successfully detected nascent $38 \mathrm{C}$ piRNA precursors that co-localized with Rhi in diploid male germ cells (Fig 6D). These signals from nascent 38C piRNA cluster transcripts were absent in rhi mutant testes, confirming the specificity of our probes (Fig 6D). We found that both $38 \mathrm{C}$ and $A T$-chX piRNA precursors can be seen in nuclei of spermatogonia. In contrast, only $A T$-chX, but not $38 \mathrm{C}$, cluster continues to be expressed in $87.7 \%(n=50 / 57)$ of early spermatocyte nuclei (Fig $6 \mathrm{E}$ ). Therefore, the expression of $38 \mathrm{C}$ piRNA cluster is turned off when Rhi concentrates onto the $A T$-chX locus.

During early spermatogenesis, satellite DNAs located at peri-centromeric heterochromatin of different chromosomes cluster together to form distinct nuclear compartments called chromocenters $[24,25]$. To explore the possibility that the repetitive $A T$-chX locus is also recruited to a chromocenter, we compared Rhi localization with that of D1, which binds the AATAT satellite on chromosome $\mathrm{X}, \mathrm{Y}$ and 4 [24]. We found that D1 does not co-localize with the single Rhi dot in early spermatocytes (Fig 6F). We also analyzed localization of Cid, a centromerespecific histone $\mathrm{H} 3$ variant that functions as an epigenetic mark for the centromere identity [26]. The single Rhi dot is present in nuclei that contain four or more separate Cid foci (Fig $6 \mathrm{G}$ ), indicating that non-homologous chromosomes are separated and occupy distinct chromosome territories in these nuclei. These results show that the AT-chX locus bound by Rhi occupies a nuclear compartment distinct from chromocenters.

Taken together, our results suggest that RDC complex binds, and thus enables, the expression of many dual-strand piRNA clusters from GSCs to spermatogonia, including 38C, but gradually concentrates onto a single locus, $A T$-chX, in early spermatocytes (Fig $6 \mathrm{H})$. Dynamic association of RDC with piRNA clusters during early spermatogenesis executes a fluid piRNA program to allow different piRNA clusters to be engaged at different developmental stages.

\section{Discussion}

Most proteins involved in piRNA pathway in Drosophila were initially identified in screens for female sterility or TE de-repression in ovaries [9,10,27-30]. However, the first described case of piRNA repression, silencing of Stellate by $\mathrm{Su}(\mathrm{Ste})$ piRNAs during spermatogenesis, indicates that piRNA pathway operates in gonads of both sexes [31,32]. Many proteins involved in piRNA pathway in ovaries are also required for male fertility and Stellate silencing in testes, supporting the conservation of piRNA pathway machinery between sexes [31,33-39]. Notably, a few proteins stood out as exceptions: Rhi, Del and Cuff that form a complex to enable transcription of dual-strand piRNA clusters in ovary $[2,4,14]$, for which no fertility defects or Stellate de-repression were observed in mutant males $[10,13,14]$. This suggested that molecular mechanisms controlling piRNA cluster expression in testis might be different from ovary. Our results, however, demonstrate that RDC complex is assembled in testis (Fig 2) and required for TE silencing (Fig 3) in male germline, indicating that the molecular machinery regulating piRNA cluster expression is conserved between sexes.

\section{piRNA pathway in Drosophila testis: beyond Stellate silencing}

Genomic loci that encode ovarian piRNAs were systematically identified across the genome in several studies $[2,40]$. In contrast, piRNA studies on testes were mostly focused on a single locus, $\mathrm{Su}(\mathrm{Ste})$, that encodes piRNAs to silence Stellate, and no systematic search for piRNA clusters in testis has been performed to date. We recently de novo identified piRNA clusters in testis [20], laying the foundation for broader understanding of piRNA biogenesis and function in male gonads. We found that RDC is essential for expression of all major dual-strand piRNA clusters in testis, with a remarkable exception of $S u(S t e)$ (Fig 4). This explains the previous 
observation that rhi and cuff are dispensable for Stellate silencing [14,15]. In contrast, many other piRNA pathway factors such as Aub, Ago3 and Zuc are involved in both Stellate and TE repression $[31,33,35]$. Mutations in these genes cause dramatic disruption of spermatogenesis, often leading to complete male sterility. In comparison, rhi, del and cuff mutant males demonstrate milder fertility defects, suggesting that Stellate de-repression is the major cause of spermatogenesis failure in other piRNA pathway mutants. Indeed, previous studies demonstrated that Stellate de-repression induced by deleting the $\mathrm{Su}(\mathrm{Ste})$ locus alone, without global perturbations of piRNA pathway, disrupts spermatogenesis and causes male sterility [41]. Thus, the finding that RDC is dispensable for Stellate repression provides a unique opportunity to understand impacts of silencing other piRNA targets in the male germline.

Our results indicate that piRNA-guided repression plays a crucial role in spermatogenesis beyond Stellate silencing, as rhi, del and cuff mutant males show rapid fertility decline, germline DNA damage and severe loss of germline content including GSCs (Fig 3). These phenotypes are likely caused by TE de-repression and the resultant genome instability, though we also identified complex satellites and a host protein-coding gene, SUMO protease CG12717/ pirate, as targets of piRNA silencing in testes [20]. In the future, it will be important to disentangle the contributions of de-repressing different piRNA targets to spermatogenesis defects observed in testes of piRNA pathway mutants.

Even though the fertility of rhi mutant males is substantially compromised, they produce a small number of functional spermatozoa, at least when they are young (Fig 1). In contrast, females lacking $r h i$, del or cuff are completely sterile $[10,13]$. This distinction between the two sexes might result from differences in the TE threat faced by male and female germ cells. Different TE families are activated upon disruption of piRNA pathway in ovary and testis and, generally, there is a stronger TE threat in ovary [20]. Differential TE de-repression in two sexes might be responsible for stronger defects in oogenesis at the absence of RDC. Alternatively, it might reflect differences in DNA damage response in two sexes. An egg is energetically more expensive to make than a spermatozoon. In line with this, DNA damage responses (activated by e.g., TE transposition) often arrest oogenesis to avoid wasting resources on a defective egg $[42,43]$. Since oogenesis is usually shut down to attempt repair, incomplete oogenesis results in female sterility. In contrast, quality control mechanisms of spermatogenesis frequently kill unqualified germ cells [44], without pausing the developmental program of those surviving ones. Because spermatogenesis permits a large number of germ cells to develop in parallel, even though the unqualified ones are killed, a few surviving germ cells might be able to complete sperm development. Together, differential TE threats coupled with distinct response strategies to DNA damage might underlie sex-specific sterility when RDC is lost.

\section{Su(Ste): an RDC-independent dual-strand piRNA cluster free of RDC binding}

$\mathrm{Su}(\mathrm{Ste})$ locus on $\mathrm{Y}$ chromosome is the most prolific source of piRNAs in testes [20]. piRNAs are generated from both genomic strands of $\mathrm{Su}(\mathrm{Ste})$ repeats, making it akin to other dualstrand piRNA clusters [31,45]. However, our results showed that RDC is dispensable for expression of $S u(S t e)$ piRNAs, while it is required for piRNA production from all other dualstrand clusters in testis (Fig 4A) and ovary [2,4]. What might explain the ability of Su(Ste) to generate piRNAs in an RDC-independent fashion? RDC ensures transcription of piRNA precursors by suppressing their premature termination [3] and promoting non-canonical transcription initiation [8]. Interestingly, the structure of $S u(S t e)$ locus is different from other dualstrand piRNA clusters, as it is composed of many almost identical, relatively short units, all of which are flanked by two canonical, albeit convergent, promoters driving expression of both 
genomic strands [31]. In fact, sense transcripts of $\mathrm{Su}(\mathrm{Ste})$ were found to be spliced and polyadenylated [31], consistent with the absence of RDC, which suppresses splicing and polyadenylation [2-4], at this locus. Thus, the presence of canonical promoters flanking individual short units of $\mathrm{Su}(\mathrm{Ste})$ repeats might enable their expression without engaging RDC complex.

Consistent with a role in promoting piRNA precursor transcription, Rhi is enriched on chromatin of dual-strand piRNA clusters. Such a correlation between direct Rhi binding and Rhi-dependent piRNA expression was previously reported in ovary [2]. The function of RDC explains why piRNA production depends on its presence on chromatin; however, the molecular mechanism responsible for specific recruitment of RDC to piRNA clusters remained poorly understood. It has been shown that piRNAs expressed during oogenesis are deposited into the oocyte and play an important role in jump-starting piRNA biogenesis in the progeny [46,47]. Maternally supplied piRNAs were shown to induce deposition of Rhi on cognate genomic locus in the progeny [7]. Thus, piRNAs expressed from the cluster and RDC binding to the cluster seem to form a positive feedback loop: RDC is required for piRNA production, and piRNAs in turn guide deposition of Rhi on cognate genomic loci. piRNA-dependent Rhi deposition might be mediated by the nuclear Piwi protein that directs the establishment of histone H3K9me3 mark [48-51], which provides a binding site for Rhi chromo-domain [2,7]. Importantly, Piwi- and piRNA-dependent Rhi recruitment seems to occur in a narrow developmental window during early embryogenesis. This was demonstrated by the observation that depleting Piwi during early embryogenesis is sufficient to perturb Rhi localization on piRNA clusters, while depleting Piwi during larval or adult stages does not change Rhi localization [52]. Our finding that Rhi is localized to all dual-strand piRNA clusters in testis except $\mathrm{Su}$ (Ste) (Fig 4D) is compatible with an idea that Rhi binding to genomic loci in the zygote is guided by maternal piRNAs. Indeed, in contrast to most other dual-strand clusters that are active in the germline of both sexes, Y-linked $\mathrm{Su}(\mathrm{Ste})$ locus generates piRNAs only in males. Therefore, unlike other piRNAs, $\mathrm{Su}(\mathrm{Ste})$ piRNAs are not deposited into the oocyte, resulting in the inability to recruit Rhi to $\mathrm{Su}(\mathrm{Ste})$ in the progeny. It is interesting to compare $\mathrm{Su}(\mathrm{Ste})$ with another dual-strand piRNA cluster on Y chromosome, petrel. Unlike Su(Ste), Rhi is enriched on petrel chromatin and piRNA production from this cluster depends on Rhi (Fig 4A and 4D). However, petrel piRNAs derived from male-specific $\mathrm{Y}$ should be absent in ovaries and hence no petrel piRNAs can be deposited into the oocyte. At the first glance, these observations argue against a possibility that maternal piRNAs guide Rhi deposition on petrel cluster. However, unlike $\mathrm{Su}(\mathrm{Ste})$, petrel is enriched of different TE sequences. As a result, TE-mapping piRNAs produced from other clusters in ovary might be able to target petrel. Indeed, piRNAs mapping to TEs present at the petrel locus can be found in unfertilized eggs [46]. For example, roo piRNAs are the most abundant TE-mapping, maternally deposited piRNAs in the early embryo [46]. Several roo fragments are present at petrel, and these sequences occupy $\sim 6 \%$ of the total length of this cluster, making roo the third most represented TE at petrel (after IDEFIX and ninja) [20]. piRNAs against other TEs located at petrel are also deposited in the embryo. Overall, while our results did not directly address the mechanism of Rhi recruitment to specific genomic loci, they show that studying Rhi occupancy on Y-linked piRNA clusters provides a novel angle to study this problem.

\section{Dynamic organization of piRNA pathway during spermatogenesis}

Our results showed that components of RDC complex are expressed exclusively in male germline during early stages of spermatogenesis, from GSCs to early spermatocytes (Figs 1 and 2). Interestingly, expression of Piwi and Ago3, two of the three PIWI proteins in Drosophila, is also restricted to the same developmental stages [34,53]. Piwi is required for piRNA-guided 
transcriptional silencing in the nucleus [48-51], while Ago3 is involved in heterotypic pingpong cycle in cytoplasm $[33,40,54]$, indicating that these processes operate in the same cells that have RDC-dependent transcription of piRNA clusters. In contrast to RDC, Piwi and Ago3, expression of the third PIWI protein, Aub, continues through spermatocyte stage until meiosis [34]. How can developing male germ cells be protected when the piRNA pathway is greatly simplified? It is possible that the silencing network initiated by piRNA pathway factors earlier can self-sustain later. piRNAs produced with the help of RDC complex and Ago3-dependent heterotypic ping-pong could persist and continue to function through spermatocyte differentiation, as long as they load onto Aub.

Interestingly, the cessation of RDC, Piwi and Ago3 expression during spermatogenesis coincides with the mitosis-to-differentiation transition. This transition is accompanied by one of the most dramatic changes in gene expression programs, with the general transcriptional machinery replaced by the testis-specific ones like tTAF and tMAC $[55,56]$. Thus, the following stage of male germline development can also be seen as a less-protected window of spermatogenesis, providing an opportunity for TEs and other selfish genetic elements to thrive.

Restriction of the piRNA pathway to early stages of spermatogenesis contrasts with its activity during oogenesis. piRNA pathway factors appear to be expressed during all stages of oogenesis from GSCs to late-stage nurse cells. Recent studies reported several new factors involved in piRNA pathway in ovary, such as Moon, Boot, Nxf3, Panx, Arx and Nxf2 [8,22,30,57-65]. These proteins are expressed at a low level in testis and their functions during spermatogenesis have not yet been reported. Our results suggest that, similar to RDC, these proteins might function in piRNA pathway in testis, and their low expression could be explained by restricted expression in early male germline. Indeed, we found that Moon co-expresses with, and acts genetically downstream of, RDC in testes (Figs 2 and S4). Overall, our results suggest that piRNA pathway machinery is likely conserved between sexes. However, the developmental organization of piRNA pathway during gametogenesis is different: whereas the entire pathway is active throughout oogenesis, processes that require RDC, Piwi or Ago3 likely terminate when mitotic spermatogonia differentiate into spermatocytes and prepare for meiosis.

\section{Dynamic expression of piRNA clusters during spermatogenesis}

Our results revealed dynamic association of RDC complex with different genomic loci during spermatogenesis (Fig 6). In GSC and spermatogonia nuclei, RDC complex localizes to many foci, and ChIP-seq data indicate that Rhi associates with multiple dual-strand clusters. As germ cells differentiate into early spermatocytes, however, RDC gradually concentrates onto a single locus, $A T$-chX, on $\mathrm{X}$ chromosome. Since transcription of dual-strand piRNA clusters is dependent on RDC complex, the dynamic localization of RDC suggests that expression of piRNA clusters changes as germ cells progress from GSCs to early spermatocytes. Indeed, detection of nascent cluster transcripts revealed that $38 \mathrm{C}$ is active early, but not later when most RDC concentrates onto AT-chX. Notably, though not dependent on Rhi, transcription of $\mathrm{Su}(\mathrm{Ste})$ piRNA cluster was shown to span a narrow window from late spermatogonia to early spermatocyte as well [45], likely reflecting the promoter activity that drives $\mathrm{Su}(\mathrm{Ste})$ transcription [31]. Dynamic expression of piRNA clusters during spermatogenesis is also supported by the study that showed spermatogonia and spermatocytes have distinct piRNA populations [66]. In contrast to dynamic localization of RDC complex and cluster expression in testes, previous work depicted a static view of piRNA production in female gonads. There has been no evidence of dynamic localization of RDC complex to different clusters during oogenesis, and all clusters appear active throughout female germline development. It will be interesting to explore whether expression of piRNA clusters changed dynamically during oogenesis. 
Through studying a protein complex thought to be absent during spermatogenesis, we uncovered the sexually dimorphic and dynamic behaviors of a molecular machinery that drives dual-strand piRNA cluster expression during Drosophila gametogenesis.

\section{Materials and methods}

\section{Fly stocks}

The following stocks were used: $b_{a m}^{\Delta 86}$ (BDSC5427), bam $^{D f}$ (BDSC27403), C(1)RM (BDSC9460), nos-Gal4 (BDSC4937), UASp-shRhi (BDSC35171), iso-1 (BDSC2057), GFP-Cid (BDSC25047), GFP-D1 (BDSC50850) were obtained from Bloomington Drosophila Stock Center; GFP-Rhi (VDRC313340), GFP-Del (VDRC313271), GFP-Cuff (VDRC313269), moon $^{\Delta 1}$ (VDRC313735), moon $^{\Delta 28}$ (VDRC313738), Bam-GFP (VDRC318001), $w^{1118}$ (VDRC60000) were obtained from Vienna Drosophila Resource Center; tj-Gal4 (DGRC104055) was obtained from Kyoto Stock Center; $r h i^{2}, r h i^{K G}$ and UASp-GFP-Rhi were gifts of William Theurkauf; del ${ }^{W K 36}$, del ${ }^{H N 56}$, cuff ${ }^{\mathrm{QQ} 37}$ and $c u f f^{W M 25}$ were gifts of Trudi Schüpbach; GFP-Vasa (gift of Paul Lasko), copiaLTR-lacZ (gift of Elena Pasyukova), GFP-Moon (gift of Peter Andersen), Sa-GFP (gift of Xin Chen). UASp-mKate2 was described before [3]. GFPtagged Rhi, Del, Cuff and Moon are previously described transgenes constructed by inserting $\mathrm{N}$-terminal GFP into genomic BACs via recombineering $[2,8]$. XO males were generated by crossing GFP-Rhi males to C(1)RM females. To perform GFP-Rhi ChIP, bam ${ }^{\Delta 86}$ and GFP-Rhi were recombined.

\section{Generation of transgenic flies}

To make mKate2-tagged Rhi driven by endogenous rhi promoter, the $\sim 2 \mathrm{~Kb}$ region upstream of $r h i$ gene that includes the putative endogenous rhi promoter [4] was cloned from genomic DNA of Drosophila melanogaster by PCR (forward primer: AGG CCT ATG TAC CAA GTT GTT AAC TCT ATC G, reverse primer: GGT ACC AGA CAT AAC TTA TCC GCT CAC AGG). PCR product was digested by Stul and Kpn1, then ligated into Stu1- and Kpn1-digested vector that contains mKate2-Rhino, mini-white gene and the $\Phi C 31$ attB site. The construct was inserted into genomic site 76A2 (y1 w1118; PBac $\{y+-a t t P-9 A\} V K 00013)$ on chromosome 3 using $\Phi C 31$-mediated recombination.

\section{Sperm exhaustion test}

The test was modified from Sun et al. (2004) and done at $25^{\circ} \mathrm{C}$. Individual 1-day old virgin males (either $r h i^{2 / K G}$ or heterozygous siblings, $\mathrm{n}=15$ ) were allowed to mate with three 4-day old wildtype virgin females (iso-1) for $24 \mathrm{hrs}$. Each male was then moved to mate with another three 4-day old wildtype virgin females (iso-1) for another $24 \mathrm{hrs}$, and this was repeated every $24 \mathrm{hrs}$ for a total of 14 days. Since each male encountered multiple young virgin females every day, their sperm were exhausted, and the number of progeny produced by females can be used to represent the daily male fertility. Inseminated females were flipped every other day and kept for 20 days (without contact with other males) to achieve maximal egg laying. A binary result of whether there is offspring or not was used to approximate whether a male is fertile or not on a given day, and we plotted the percentage of fertile male out of 15 tested males each day for $r h i^{2 / K G}$ and heterozygous siblings. To probe the male fertility more quantitatively, we repeated the test with five males for ten days. Instead of recording a binary result, we counted the number of progeny. The number of adult offspring was counted 15 days after female fly removal to allow most laid eggs to develop into adulthood. The averaged total number of offspring for each male each day was plotted for each genotype. This was repeated for $\mathrm{del}^{\mathrm{HN} / W K}, \mathrm{cuff}^{\mathrm{WM} 25 /}$ 
${ }^{Q Q 37}$ and respective heterozygous controls ( $\mathrm{n}=5$ per genotype), where each 1-day-old virgin male was mated with two 4 -day-old $w^{1118}$ virgin females every day for a total of eleven days. After male removal, inseminated females were flipped every 3 days for a total of 15 days for counting.

\section{Female fertility test}

Each 1-day-old virgin female with the genotype of interest was allowed to mate with two 5 -day-old $w^{1118}$ males for four days. Next, flies were discarded but vials were kept for another 14 days before counting, so eggs laid had 14-18 days to develop to adulthood. The number of adult flies from each vial was counted to approximate the female fertility. For each of the four groups shown in S2 Fig, all three genotypes (control, mutant and rescue) were siblings from the same cross with similar genetic backgrounds. Results were obtained from three biological replicates $(\mathrm{n}=3)$.

\section{Immunofluorescence staining}

Testes were dissected in PBS, fixed in $4 \%$ formaldehyde for $20 \mathrm{mins}$ and washed by PBSTw (PBS with 0.1\% Tween-20) for 3 times. Permeabilization of testes was done by incubation with PBST (PBS with $0.5 \%$ Triton-X) for 30 mins. Testes were then blocked by $5 \%$ BSA in PBSTw for at least an hour, before incubation with primary antibody in 5\% BSA in PBSTw at $4^{\circ} \mathrm{C}$ overnight. Testes were washed 3 times with PBSTw and incubated with secondary antibody in 5\% BSA in PBSTw at room temperature for $2 \mathrm{hrs}$, followed by another 3 washes with PBSTw. Before mounting in VECTA-SHIELD, testes were stained by DAPI (1:5000) for 10mins and rinsed once with PBS. The following primary antibodies were used: mouse anti-Fas3 (7G10, 1:200), mouse anti- $\gamma \mathrm{H} 2 \mathrm{Av}$ (UNC93-5.2.1, 1:400) and rat anti-Vasa (concentrated, 1:100) were obtained from Developmental Studies Hybridoma Bank.

\section{RNA fluorescence in situ hybridization (RNA FISH)}

RNA FISH was done as described previously [67]. Fixed testes were prepared as above for immunofluorescence staining. Permeabilization was done by incubation in PBST with $0.5 \%$ sodium deoxycholate for an hour, followed by 3 washes of PBSTw. Testes were transferred to first $25 \%$ and then $50 \%$ formamide, both for $10 \mathrm{mins}$. Next, testes were prehybridized in hybridization buffer ( $50 \%$ formamide, $0.5 \mathrm{mg} / \mathrm{ml}$ yeast tRNA, $0.2 \mathrm{mg} / \mathrm{ml}$ heparin) for $1 \mathrm{hr}$ at $42^{\circ} \mathrm{C}$, before incubation with $0.1-1 \mu \mathrm{g}$ DIG-labeled RNA probe in $50 \mu$ l hybridization buffer overnight at $42^{\circ} \mathrm{C}$ with shaking. Testes were rinsed twice with $50 \%$ formamide for $20 \mathrm{mins}$ at $42^{\circ} \mathrm{C}$ and then transferred to wash in PBSTw for 4 times. Subsequent blocking, staining by sheep antiDIG antibody (PA1-85378, 1:200, Life Technologies) and mounting were the same as described above for immunofluorescence staining. DIG-labeled RNA probes were transcribed by T7 according to manufacturer's instructions. DNA template was made from genomic PCR using primers listed below, with $\mathrm{T} 7$ promoter sequence added 5 ' to the reverse primers.

$\mathrm{Su}(\mathrm{Ste})[45]$

F: 5'-CAGGTGATTACCACTATTAACGAAAAGTATGC

R: 5'-ATCCTCGGCCAGCTAGTCCT

$A T-\operatorname{ch} X[67]$

F: 5'-AGCGATCCCACTGCTAAAGA

R: 5'ATAAAAGGTGACCG-GCAACG 


\section{RNA in situ hybridization chain reaction (HCR)}

A kit containing a DNA probe set, a DNA probe amplifier and hybridization, amplification and wash buffers were purchased from Molecular Instruments (molecularinstruments.org) for $A T$-ch $X$ and $38 C$ transcripts. To minimize off-targets, we designed probes targeting unique regions at $A T$-chX and $38 C$. For $38 C$, we specifically targeted junction sites of two different TEs, the simultaneous presence of which is required to generate amplified HCR signals. The AT-chX (unique identifier: 3893/E038) and 38C (unique identifier: 4026/E138-E140) probe sets initiated B1 (Alexa 647) and B3 (Alexa 546) amplifiers, respectively. In situ HCR v3.0 [23] was performed according to manufacturer's recommendations for generic samples in solution.

\section{X-gal staining}

Testes were dissected in PBS, fixed in $0.5 \%$ glutaraldehyde containing $1 \mathrm{mM} \mathrm{MgCl}_{2}$ for 5 mins and washed twice in PBS. Testes were incubated with $0.02 \% \mathrm{X}$-gal in X-gal buffer $(1 \mathrm{mM}$ $\mathrm{MgCl}_{2}, 150 \mathrm{mM} \mathrm{NaCl}, 10 \mathrm{mM} \mathrm{Na}_{2} \mathrm{HPO}_{4}, 10 \mathrm{mM} \mathrm{NaH}_{2} \mathrm{PO}_{4}, 3.5 \mathrm{mM} \mathrm{K}_{4} \mathrm{Fe}(\mathrm{CN})_{6}$ and $3.5 \mathrm{mM}$ $\left.\mathrm{K}_{3} \mathrm{Fe}(\mathrm{CN})_{6}\right)$ at $37^{\circ} \mathrm{C}$ in dark for time of interest. Staining of copiaLTR-lacZ in $r h i^{2 / K G}$, del ${ }^{H N / W K}$ and cuff $^{W M 25 / \mathrm{QQ} 37}$ typically took $1.5-2.5 \mathrm{hrs}$ to develop. Reaction was then stopped by two washes of PBS and mounted as above for RNA FISH.

\section{Image acquisition and analysis}

Images were acquired using confocal microscope Zeiss LSM 800 with 63x oil immersion objective $(\mathrm{NA}=1.4)$ and processed using the software Fiji [68]. X-gal stained testes were imaged with 10x objective $(\mathrm{NA}=0.3)$. Maximum-intensity z-projection was done in Fiji, and line intensity profiles were obtained in Fiji. All images shown were from single focal planes, unless otherwise stated. Dotted outlines were drawn for illustration purposes. To quantify the number of GSCs, we stained the hub by Fas3. Z-stacks were acquired with $0.5 \mu \mathrm{m}$ intervals to cover depths well above and below the entire hub. Vasa-positive germ cells directly adjacent to the hub in 3D were deemed as GSCs and manually counted for each testis. Even though a molecular marker for GSCs was not used, any bias in GSC counting should be shared by both $r h i^{2 / K G}$ and heterozygous sibling controls.

\section{RNA-seq and analysis}

RNA was extracted from dissected testes of $0-3$ days old $r h i^{2 / K G}$ and heterozygous sibling controls using TRIzol (Invitrogen). About $1 \mu \mathrm{g}$ RNA for each sample was subject to polyA+ selection using NEBNext Poly(A) mRNA Magnetic Isolation Module (NEB E7490) and then strand-specific library prep using NEBNext Ultra Directional RNA Library Prep Kit for Illumina (NEB E7760) according to manufacturer's instructions. For each genotype, two biological replicates were sequenced on Illumina HiSeq 2500 yielding 25-33 million 50bp single-end reads. Reads mapped to $D$. mel rRNA were discarded by bowtie 1.2.2 allowing 3 mismatches ( $<2 \%$ across all polyA-selected samples). For TE analysis, rRNA-depleted reads were mapped to TE consensus from RepBase17.08 using bowtie 1.2.2 with -v 3 -k 1. Mapped reads were normalized to the total number of reads that can be mapped to $\mathrm{dm} 6$ genome. Consistency between biological replicates was confirmed by $>0.98$ correlation coefficient, so the mean of them was used for all analyses. For simplicity, reads mapped to LTR and internal sequence were merged for each LTR TE given their well correlated behaviors. Note that polyA-selection was done for TE quantification in order to exclude piRNA precursor transcripts from dual-strand clusters and non-canonical transcripts from individual TEs, which are not polyadenylated but share sequence homology with TEs. 


\section{piRNA-seq and analysis}

RNA extraction was done as above for RNA-seq. 19-30nt small RNAs were purified by PAGE (15\% polyacrylamide gel) from $\sim 1 \mu \mathrm{g}$ total RNA. Purified small RNA was subject to library prep using NEBNext Multiplex Small RNA Sample Prep Set for Illumina (NEB E7330) according to manufacturer's instructions. Adaptor-ligated, reverse-transcribed, PCR-amplified samples were purified again by PAGE (6\% polyacrylamide gel). Two biological replicates per genotype were sequenced on Illumina HiSeq 2500 yielding 15-20 million 50bp single-end reads. Adaptors were trimmed with cutadapt 2.5 and size-selected for 23-29nt sequences for piRNA analysis. 23-29nt reads that mapped to rRNA were discarded by bowtie 1.2.2 tolerating 3 mismatches ( $<30 \%$ in control samples). For TE analysis, 23-29nt small RNA reads were mapped and normalized as done for polyA+ RNA described above, with correlation coefficients between replicates all $>0.94$. Averages of two well-correlated replicates were used for all analyses. Complex satellite-mapping small RNAs were analyzed similarly (with ovary data downloaded from GSE126578). For piRNA cluster analysis, we used piRNA clusters defined in [20]. $1 \mathrm{~Kb}$ genomic windows in individual piRNA clusters were generated with bedtools v2.28.0, and the ones including highly expressed miRNA, snRNA, snoRNA, hpRNA or 7SL SRP RNA were excluded. Coverage over individual piRNA clusters were computed using the pipeline tolerating local repeats described in [20]. A pseudo-count of 1 was added before calculating $\log 2$ fold-change of rhi mutant over control.

\section{ChIP-qPCR, ChIP-seq and analysis}

ChIP protocol was modified based on Le Thomas et al. (2014). For each biological replicate, 200 pairs of 0-2 days old testes or 100 pairs of 4-5 days old ovaries (yeast-fed for 3 days) were fixed in $1 \%$ formaldehyde for $10 \mathrm{mins}$, quenched by $25 \mathrm{mM}$ glycine for $5 \mathrm{mins}$ and washed 3 times with PBS. Fixed testes were snap-frozen in liquid nitrogen and stored at $-80^{\circ} \mathrm{C}$ before ChIP. Frozen testes were first resuspended in PBS and then washed in Farnham Buffer $(5 \mathrm{mM}$ HEPES pH8.0, 85mM KCl, 0.5\% NP-40, protease inhibitor, $10 \mathrm{mM} \mathrm{NaF}, 0.2 \mathrm{mM} \mathrm{Na}_{3} \mathrm{VO}_{4}$ ) twice. Testes were then homogenized in RIPA Buffer (20mM Tris pH7.4, $150 \mathrm{mM} \mathrm{NaCl}, 1 \%$ NP- $40,0.5 \%$ sodium deoxycholate, $0.1 \%$ SDS, protease inhibitor, $10 \mathrm{mM} \mathrm{NaF}, 0.2 \mathrm{mM}$ $\mathrm{Na}_{3} \mathrm{VO}_{4}$ ) using a glass douncer and a tight pestle. Sonication was done in Bioruptor (Diagenode) on high power for 25 cycles (30s on and 30s off). Sonicated tissues were centrifugated to obtain the supernatant. The supernatant was pre-cleared with Dynabeads Protein G beads (Invitrogen) for $2 \mathrm{hrs}$ at $4^{\circ} \mathrm{C} .5 \%$ of the pre-cleared sample was set aside as the Input, while the rest was incubated with $5 \mu \mathrm{l}$ anti-GFP antibody (A-11122, Invitrogen) overnight at $4^{\circ} \mathrm{C}$. The immune-precipitated (IP) sample was incubated with Dynabeads Protein G beads for $5 \mathrm{hrs}$ at $4^{\circ} \mathrm{C}$ to allow beads binding. After that, beads were washed 5 times in $\mathrm{LiCl}$ Wash Buffer $(10 \mathrm{mM}$ Tris pH7.4, 500mM LiCl, $1 \%$ NP-40, $1 \%$ sodium deoxycholate), while the Input sample was incubated with $1 \mu \mathrm{l} 10 \mathrm{mg} / \mathrm{ml}$ RNase A at $37^{\circ} \mathrm{C}$ for $1 \mathrm{hr}$. Both IP and Input samples were incu-

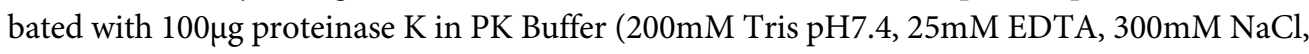
$2 \% \mathrm{SDS}$ ) first at $55^{\circ} \mathrm{C}$ for $3 \mathrm{hrs}$ and then at $65^{\circ} \mathrm{C}$ overnight. DNA was purified by phenol-chloroform extraction and the concentration was measured by Qubit. Four biological replicates of testis $\left(\mathrm{bam}^{\Delta 86 / D f}\right.$, GFP-Rhi) ChIP were done and used in qPCR, with two randomly selected replicates sequenced. Two biological replicates of ovary ChIP (GFP-Rhi) were done and sequenced. ChIP-qPCR was normalized first to Input and then to a negative control region (free of Rhi binding in ovary according to Mohn et al. 2014) to obtain Rhi enrichment (primers listed below). ChIP DNA was subject to library prep using NEBNext ChIP-Seq Library Prep Master Mix Set for Illumina (NEB E6240) according to manufacturer's instructions. Two biological replicates per sex were sequenced on Illumina HiSeq 2500 yielding 13-22 million 
50bp single-end reads. Reads were mapped to the genome as described in [20] permitting local repeats. Coverage over piRNA clusters were computed and the enrichment of IP over input was calculated. Two biological replicates were consistent with a correlation coefficient $>0.96$, so the average enrichment was plotted.

$42 A B[14]$

F: 5'-GTG GAG TTT GGT GCA GAA GC

R: 5'-AGC CGT GCT TTA TGC TTT AC

flam [14]

F: 5'-TGA GGA ATG AAT CGC TTT GAA

R: 5'-TGG TGA AAT ACC AAA GTC TTG GGT CAA

$\mathrm{Su}(\mathrm{Ste})[31]$

F: 5'-CTTGGACCGAACACTTTGAACCAAGTATT

R: 5'-GGCATGATTCACGCCCGATACAT

$A T-\operatorname{ch} X[67]$

F: 5'-AGCGATCCCACTGCTAAAGA

R: 5'-GTCGAAGACGTCCAGAGGAG

negative control for Rhi binding (this study)

F: 5'AAGAGCAGAGGGGCCAAATC

R: 5'-TCCAAGTCGGCTTCCCTTTC

\section{Genome-wide relationship between piRNA production and Rhi binding}

This analysis was adapted from Mohn et al. (2014) with modifications. piRNA production and Rhi enrichment were computed for individual $1 \mathrm{~Kb}$ windows tiling the entire $\mathrm{dm} 6$ genome. The average of two well-correlative biological replicates was used for this analysis. Only $1 \mathrm{~Kb}$ windows having both $\geq 4 \mathrm{RPM}$ piRNAs in controls and $\geq 30 \mathrm{RPM}$ reads in IP samples were plotted. Rhi-dependent loci ( $R D$ loci) were defined as $1 \mathrm{~Kb}$ windows showing $\geq 4$-fold drop in piRNA production in rhi mutant testes, and the rest were treated as Rhi-independent loci (RI loci). Local regression was implemented with LOESS technique in python.

\section{Data visualization and statistical analysis}

Most data visualization and statistical analysis were done in Python 3 via JupyterLab using the following software packages: numpy [69], pandas [70] and altair [71]. Germ cell death, GSC loss and ChIP-qPCR were plotted in GraphPad Prism. Mann-Whitney-Wilcoxon test was done to compute $\mathrm{p}$ values for GSC loss. Multiple t-tests corrected for multiple comparisons by the Holm-Sidak method were done for Rhi ChIP-qPCR, using the uni-strand cluster flam known to be free of Rhi binding as a negative control. The UCSC Genome Brower [72] and IGV $[73,74]$ were used to conduct explorative analysis of sequencing data.

\section{Supporting information}

S1 Fig. Del and Cuff are required for normal male fertility. Compromised fertility of del (top) and cuff (bottom) mutant males. Sperm exhaustion test of $\mathrm{del}^{\mathrm{HN} / W K}$ or cuff $\mathrm{WM25/QQ37}^{\mathrm{W}}$ 
mutant (orange) and respective heterozygous sibling control (blue) males. Left: averaged numbers of offspring per male 1-11 days after eclosion $(n=5)$. Right: total number of progeny per male after mating for 11 days. Shaded areas and error bars display standard error. $P$ value from unpaired t-test.

S2 Fig. Rescue of female sterility by transgenes used in this study. GFP-tagged Rhi, Del and Cuff transgenes as well as mKate2-tagged Rhi transgene driven by a putative rhi promoter fully rescue the female sterility of respective mutations. (TIF)

S3 Fig. Characterization of the expression of Rhi in testis. Individual channels of images shown in Fig 2D.

S4 Fig. Moon acts downstream of Rhi in testis. Confocal images of apical tips of testes expressing GFP-Rhi, in moon $^{\Delta 1}$ and moon $^{\Delta 28}$ mutant backgrounds. Note that moon is Xlinked, so XY males only have one copy of moon and trans-heterozygous mutant cannot be generated.

S5 Fig. Production of TE-antisense and TE-sense piRNAs collapses without rhi. Loss of TEmapping piRNAs in testes of rhi mutants. Scatter plot showing expression of TE-antisense (left) and TE-sense (right) piRNAs in $r h i^{2 / K G}$ mutant versus heterozygous control testes. piRNAs that show $\geq 2$-fold reduction (FDR $<0.05)$ and $\geq 10$ RPM average expression levels are marked in blue. Shown are averages of two biological replicates.

(TIF)

\section{Acknowledgments}

We are grateful to Xin Chen, Peter Andersen, William Theurkauf, Trudi Schüpbach, Paul Lasko, Elena Pasyukova and three Drosophila Stock Centers (Bloomington, Vienna, Kyoto) for fly stocks. We thank Katalin Fejes Toth and members of Aravin lab for discussion and comments. We appreciate the help of Maria Ninova and Fan Gao (Bioinformatics Resource Center, Caltech) with bioinformatics analysis, the help of Grace Shin and Maayan Schwarzkopf with HCR experiments, the help of Giada Spigolon and Andres Collazo (Biological Imaging Facility, Caltech) with microscopy, and the help of Igor Antoshechkin (Millard and Muriel Jacobs Genetics and Genomics Laboratory, Caltech) with sequencing.

\section{Author Contributions}

Conceptualization: Peiwei Chen, Alexei A. Aravin.

Data curation: Peiwei Chen, Alexei A. Aravin.

Formal analysis: Peiwei Chen, Alexei A. Aravin.

Funding acquisition: Alexei A. Aravin.

Investigation: Peiwei Chen, Yicheng Luo.

Methodology: Peiwei Chen, Alexei A. Aravin.

Project administration: Peiwei Chen, Alexei A. Aravin.

Resources: Peiwei Chen, Alexei A. Aravin. 
Software: Peiwei Chen, Alexei A. Aravin.

Supervision: Peiwei Chen, Alexei A. Aravin.

Validation: Peiwei Chen, Yicheng Luo, Alexei A. Aravin.

Visualization: Peiwei Chen, Alexei A. Aravin.

Writing - original draft: Peiwei Chen, Alexei A. Aravin.

Writing - review \& editing: Peiwei Chen, Alexei A. Aravin.

\section{References}

1. Ozata DM, Gainetdinov I, Zoch A, O'Carroll D, Zamore PD. PIWI-interacting RNAs: small RNAs with big functions. Nat Rev Genet. 2019; 20: 89-108. https://doi.org/10.1038/s41576-018-0073-3 PMID: 30446728

2. Mohn F, Sienski G, Handler D, Brennecke J. The Rhino-Deadlock-Cutoff Complex Licenses Noncanonical Transcription of Dual-Strand piRNA Clusters in Drosophila. Cell. 2014; 157: 1364-1379. https://doi. org/10.1016/j.cell.2014.04.031 PMID: 24906153

3. Chen Y-CA, Stuwe E, Luo Y, Ninova M, Le Thomas A, Rozhavskaya E, et al. Cutoff Suppresses RNA Polymerase II Termination to Ensure Expression of piRNA Precursors. Molecular Cell. 2016; 63: 97109. https://doi.org/10.1016/j.molcel.2016.05.010 PMID: 27292797

4. Zhang Z, Wang J, Schultz N, Zhang F, Parhad SS, Tu S, et al. The HP1 Homolog Rhino Anchors a Nuclear Complex that Suppresses piRNA Precursor Splicing. Cell. 2014; 157: 1353-1363. https://doi. org/10.1016/j.cell.2014.04.030 PMID: 24906152

5. Rangan $P$, Malone CD, Navarro $C$, Newbold SP, Hayes PS, Sachidanandam R, et al. piRNA Production Requires Heterochromatin Formation in Drosophila. Current Biology. 2011; 21: 1373-1379. https://doi. org/10.1016/j.cub.2011.06.057 PMID: 21820311

6. Chang TH, Mattei E, Gainetdinov I, Colpan C, Weng Z, Zamore PD. Maelstrom Represses Canonical Polymerase II Transcription within Bi-directional piRNA Clusters in Drosophila melanogaster. Molecular Cell. 2019; 73: 291-303.e6. https://doi.org/10.1016/j.molcel.2018.10.038 PMID: 30527661

7. Le Thomas A, Stuwe E, Li S, Du J, Marinov G, Rozhkov N, et al. Transgenerationally inherited piRNAs trigger piRNA biogenesis by changing the chromatin of piRNA clusters and inducing precursor processing. Genes Dev. 2014; 28: 1667-1680. https://doi.org/10.1101/gad.245514.114 PMID: 25085419

8. Andersen PR, Tirian L, Vunjak M, Brennecke J. A heterochromatin-dependent transcription machinery drives piRNA expression. Nature. 2017; 549: 54-59. https://doi.org/10.1038/nature23482 PMID: 28847004

9. Berg CA, Spradling AC. Studies on the rate and site-specificity of $P$ element transposition. Genetics. 1991; 127: 515-524. PMID: 1849859

10. Schüpbach T, Wieschaus E. Female sterile mutations on the second chromosome of Drosophila melanogaster. II. Mutations blocking oogenesis or altering egg morphology. Genetics. 1991; 129: 11191136. PMID: 1783295

11. Brown JB, Boley N, Eisman R, May GE, Stoiber MH, Duff MO, et al. Diversity and dynamics of the Drosophila transcriptome. Nature. 2014; 512: 393-399. https://doi.org/10.1038/nature12962 PMID: 24670639

12. Vermaak D, Henikoff S, Malik HS. Positive selection drives the evolution of rhino, a member of the heterochromatin protein 1 family in Drosophila. PLoS Genet. 2005; 1: 96-108. https://doi.org/10.1371/ journal.pgen.0010009 PMID: 16103923

13. Volpe AM, Horowitz H, Grafer CM, Jackson SM, Berg CA. Drosophila rhino encodes a female-specific chromo-domain protein that affects chromosome structure and egg polarity. Genetics. 2001; 159: 1117-1134. PMID: 11729157

14. Klattenhoff C, Xi H, Li C, Lee S, Xu J, Khurana JS, et al. The Drosophila HP1 Homolog Rhino Is Required for Transposon Silencing and piRNA Production by Dual-Strand Clusters. Cell. 2009; 138: 1137-1149. https://doi.org/10.1016/j.cell.2009.07.014 PMID: 19732946

15. Kibanov MV, Egorova KS, Ryazansky SS, Sokolova OA, Kotov AA, Olenkina OM, et al. A novel organelle, the piNG-body, in the nuage of Drosophila male germ cells is associated with piRNA-mediated gene silencing. Brill JA, editor. MBoC. 2011; 22: 3410-3419. https://doi.org/10.1091/mbc.E11-02-0168 PMID: 21775629

16. Sun S, Ting C-T, Wu C-I. The normal function of a speciation gene, Odysseus, and its hybrid sterility effect. Science. 2004; 305: 81-83. https://doi.org/10.1126/science.1093904 PMID: 15232104 
17. Vermaak D, Malik HS. Multiple roles for heterochromatin protein 1 genes in Drosophila. Annu Rev Genet. 2009; 43: 467-492. https://doi.org/10.1146/annurev-genet-102108-134802 PMID: 19919324

18. Kalmykova AI, Klenov MS, Gvozdev VA. Argonaute protein PIWI controls mobilization of retrotransposons in the Drosophila male germline. Nucleic Acids Res. 2005; 33: 2052-2059. https://doi.org/10. 1093/nar/gki323 PMID: 15817569

19. Pasyukova E, Nuzhdin S, Li W, Flavell AJ. Germ line transposition of the copia retrotransposon in Drosophila melanogaster is restricted to males by tissue-specific control of copia RNA levels. Mol Gen Genet. 1997; 255: 115-124. https://doi.org/10.1007/s004380050479 PMID: 9230904

20. Chen P, Kotov AA, Godneeva BK, Bazylev SS, Olenina LV, Aravin AA. piRNA-mediated gene regulation and adaptation to sex-specific transposon expression in $D$. melanogaster male germline. Genes Dev. 2021; 35:914-935. https://doi.org/10.1101/gad.345041.120 PMID: 33985970

21. Vazquez J, Belmont AS, Sedat JW. The dynamics of homologous chromosome pairing during male Drosophila meiosis. Curr Biol. 2002; 12: 1473-1483. https://doi.org/10.1016/s0960-9822(02)01090-4 PMID: 12225662

22. EIMaghraby MF, Andersen PR, Pühringer F, Hohmann U, Meixner K, Lendl T, et al. A Heterochromatin-Specific RNA Export Pathway Facilitates piRNA Production. Cell. 2019; 178: 964-979.e20. https:// doi.org/10.1016/j.cell.2019.07.007 PMID: 31398345

23. Choi HMT, Schwarzkopf M, Fornace ME, Acharya A, Artavanis G, Stegmaier J, et al. Third-generation in situ hybridization chain reaction: multiplexed, quantitative, sensitive, versatile, robust. Development. 2018;145. https://doi.org/10.1242/dev.165753 PMID: 29945988

24. Jagannathan $M$, Cummings $R$, Yamashita $Y M$. A conserved function for pericentromeric satellite DNA. Elife. 2018;7. https://doi.org/10.7554/eLife.34122 PMID: 29578410

25. Jagannathan $M$, Cummings $R$, Yamashita $Y M$. The modular mechanism of chromocenter formation in Drosophila. eLife. 2019; 8: e43938. https://doi.org/10.7554/eLife.43938 PMID: 30741633

26. Henikoff S, Ahmad K, Platero JS, van Steensel B. Heterochromatic deposition of centromeric histone H3-like proteins. Proc Natl Acad Sci U S A. 2000; 97: 716-721. https://doi.org/10.1073/pnas.97.2.716 PMID: 10639145

27. Czech B, Preall JB, McGinn J, Hannon GJ. A Transcriptome-wide RNAi Screen in the Drosophila Ovary Reveals Factors of the Germline piRNA Pathway. Molecular Cell. 2013; 50: 749-761. https://doi.org/10. 1016/j.molcel.2013.04.007 PMID: 23665227

28. Handler D, Meixner K, Pizka M, Lauss K, Schmied C, Gruber FS, et al. The Genetic Makeup of the Drosophila piRNA Pathway. Molecular Cell. 2013; 50: 762-777. https://doi.org/10.1016/j.molcel.2013.04. 031 PMID: 23665231

29. Karpen GH, Spradling AC. Analysis of subtelomeric heterochromatin in the Drosophila minichromosome Dp1187 by single P element insertional mutagenesis. Genetics. 1992; 132: 737-753. PMID: 1334894

30. Muerdter F, Guzzardo PM, Gillis J, Luo Y, Yu Y, Chen C, et al. A Genome-wide RNAi Screen Draws a Genetic Framework for Transposon Control and Primary piRNA Biogenesis in Drosophila. Molecular Cell. 2013; 50: 736-748. https://doi.org/10.1016/j.molcel.2013.04.006 PMID: 23665228

31. Aravin AA, Naumova NM, Tulin AV, Vagin VV, Rozovsky YM, Gvozdev VA. Double-stranded RNAmediated silencing of genomic tandem repeats and transposable elements in the D. melanogaster germline. Current Biology. 2001; 11: 1017-1027. https://doi.org/10.1016/s0960-9822(01)00299-8 PMID: 11470406

32. Vagin VV, Sigova A, Li C, Seitz H, Gvozdev V, Zamore PD. A distinct small RNA pathway silences selfish genetic elements in the germline. Science. 2006; 313: 320-324. https://doi.org/10.1126/science. 1129333 PMID: 16809489

33. Li C, Vagin VV, Lee S, Xu J, Ma S, Xi H, et al. Collapse of Germline piRNAs in the Absence of Argonaute3 Reveals Somatic piRNAs in Flies. Cell. 2009; 137: 509-521. https://doi.org/10.1016/j.cell.2009. 04.027 PMID: 19395009

34. Nagao A, Mituyama $T$, Huang $H$, Chen $D$, Siomi MC, Siomi $H$. Biogenesis pathways of piRNAs loaded onto AGO3 in the Drosophila testis. RNA. 2010; 16: 2503-2515. https://doi.org/10.1261/rna.2270710 PMID: 20980675

35. Pane A, Wehr K, Schüpbach T. zucchini and squash Encode Two Putative Nucleases Required for rasiRNA Production in the Drosophila Germline. Developmental Cell. 2007; 12: 851-862. https://doi. org/10.1016/j.devcel.2007.03.022 PMID: 17543859

36. Schmidt A, Palumbo G, Bozzetti MP, Tritto P, Pimpinelli S, Schäfer U. Genetic and molecular characterization of sting, a gene involved in crystal formation and meiotic drive in the male germ line of Drosophila melanogaster. Genetics. 1999; 151: 749-760. PMID: 9927466 
37. Stapleton W, Das S, McKee BD. A role of the Drosophila homeless gene in repression of Stellate in male meiosis. Chromosoma. 2001; 110: 228-240. https://doi.org/10.1007/s004120100136 PMID: 11513298

38. Tomari Y, Du T, Haley B, Schwarz DS, Bennett R, Cook HA, et al. RISC assembly defects in the Drosophila RNAi mutant armitage. Cell. 2004; 116: 831-841. https://doi.org/10.1016/s0092-8674(04) 00218-1 PMID: 15035985

39. Zhang Z, Xu J, Koppetsch BS, Wang J, Tipping C, Ma S, et al. Heterotypic piRNA Ping-Pong requires qin, a protein with both E3 ligase and Tudor domains. Mol Cell. 2011; 44: 572-584. https://doi.org/10. 1016/j.molcel.2011.10.011 PMID: 22099305

40. Brennecke J, Aravin AA, Stark A, Dus M, Kellis M, Sachidanandam R, et al. Discrete Small RNA-Generating Loci as Master Regulators of Transposon Activity in Drosophila. Cell. 2007; 128: 1089-1103. https://doi.org/10.1016/j.cell.2007.01.043 PMID: 17346786

41. Hardy RW, Lindsley DL, Livak KJ, Lewis B, Siversten AL, Joslyn GL, et al. Cytogenetic analysis of a segment of the $Y$ chromosome of Drosophila melanogaster. Genetics. 1984; 107: 591-610. PMID: 6430748

42. Chen $\mathrm{Y}, \mathrm{Pane} \mathrm{A}$, Schüpbach $\mathrm{T}$. Cutoff and aubergine mutations result in retrotransposon upregulation and checkpoint activation in Drosophila. Curr Biol. 2007; 17: 637-642. https://doi.org/10.1016/j.cub. 2007.02.027 PMID: 17363252

43. Klattenhoff C, Bratu DP, McGinnis-Schultz N, Koppetsch BS, Cook HA, Theurkauf WE. Drosophila rasiRNA pathway mutations disrupt embryonic axis specification through activation of an ATR/Chk2 DNA damage response. Dev Cell. 2007; 12: 45-55. https://doi.org/10.1016/j.devcel.2006.12.001 PMID: 17199040

44. Yacobi-Sharon $\mathrm{K}$, Namdar $\mathrm{Y}$, Arama E. Alternative germ cell death pathway in Drosophila involves HtrA2/Omi, lysosomes, and a caspase-9 counterpart. Dev Cell. 2013; 25: 29-42. https://doi.org/10. 1016/j.devcel.2013.02.002 PMID: 23523076

45. Aravin AA, Klenov MS, Vagin VV, Bantignies F, Cavalli G, Gvozdev VA. Dissection of a Natural RNA Silencing Process in the Drosophila melanogaster Germ Line. MCB. 2004; 24: 6742-6750. https://doi. org/10.1128/MCB.24.15.6742-6750.2004 PMID: 15254241

46. Brennecke J, Malone CD, Aravin AA, Sachidanandam R, Stark A, Hannon GJ. An epigenetic role for maternally inherited piRNAs in transposon silencing. Science. 2008; 322: 1387-1392. https://doi.org/ 10.1126/science.1165171 PMID: 19039138

47. de Vanssay A, Bougé A-L, Boivin A, Hermant C, Teysset L, Delmarre V, et al. Paramutation in Drosophila linked to emergence of a piRNA-producing locus. Nature. 2012; 490: 112-115. https://doi.org/10. 1038/nature11416 PMID: 22922650

48. Le Thomas A, Rogers AK, Webster A, Marinov GK, Liao SE, Perkins EM, et al. Piwi induces piRNAguided transcriptional silencing and establishment of a repressive chromatin state. Genes Dev. 2013; 27: 390-399. https://doi.org/10.1101/gad.209841.112 PMID: 23392610

49. Rozhkov NV, Hammell M, Hannon GJ. Multiple roles for Piwi in silencing Drosophila transposons. Genes Dev. 2013; 27: 400-412. https://doi.org/10.1101/gad.209767.112 PMID: 23392609

50. Sienski G, Dönertas D, Brennecke J. Transcriptional silencing of transposons by Piwi and maelstrom and its impact on chromatin state and gene expression. Cell. 2012; 151: 964-980. https://doi.org/10. 1016/j.cell.2012.10.040 PMID: 23159368

51. Wang SH, Elgin SCR. Drosophila Piwi functions downstream of piRNA production mediating a chromatin-based transposon silencing mechanism in female germ line. Proc Natl Acad Sci USA. 2011; 108: 21164-21169. https://doi.org/10.1073/pnas.1107892109 PMID: 22160707

52. Akkouche A, Mugat B, Barckmann B, Varela-Chavez C, Li B, Raffel R, et al. Piwi Is Required during Drosophila Embryogenesis to License Dual-Strand piRNA Clusters for Transposon Repression in Adult Ovaries. Mol Cell. 2017; 66: 411-419.e4. https://doi.org/10.1016/j.molcel.2017.03.017 PMID: 28457744

53. Cox DN, Chao A, Lin $\mathrm{H}$. piwi encodes a nucleoplasmic factor whose activity modulates the number and division rate of germline stem cells. Development. 2000; 127: 503-514. PMID: 10631171

54. Malone CD, Brennecke J, Dus M, Stark A, McCombie WR, Sachidanandam R, et al. Specialized piRNA Pathways Act in Germline and Somatic Tissues of the Drosophila Ovary. Cell. 2009; 137: 522-535. https://doi.org/10.1016/j.cell.2009.03.040 PMID: 19395010

55. Beall EL, Lewis PW, Bell M, Rocha M, Jones DL, Botchan MR. Discovery of tMAC: a Drosophila testisspecific meiotic arrest complex paralogous to Myb-Muv B. Genes Dev. 2007; 21: 904-919. https://doi. org/10.1101/gad.1516607 PMID: 17403774 
56. Hiller M, Chen X, Pringle MJ, Suchorolski M, Sancak Y, Viswanathan S, et al. Testis-specific TAF homologs collaborate to control a tissue-specific transcription program. Development. 2004; 131: 52975308. https://doi.org/10.1242/dev.01314 PMID: 15456720

57. Batki J, Schnabl J, Wang J, Handler D, Andreev VI, Stieger CE, et al. The nascent RNA binding complex SFiNX licenses piRNA-guided heterochromatin formation. Nat Struct Mol Biol. 2019; 26: 720-731. https://doi.org/10.1038/s41594-019-0270-6 PMID: 31384064

58. Dönertas D, Sienski G, Brennecke J. Drosophila Gtsf1 is an essential component of the Piwi-mediated transcriptional silencing complex. Genes Dev. 2013; 27: 1693-1705. https://doi.org/10.1101/gad. 221150.113 PMID: 23913922

59. Fabry MH, Ciabrelli F, Munafò M, Eastwood EL, Kneuss E, Falciatori I, et al. piRNA-guided co-transcriptional silencing coopts nuclear export factors. Elife. 2019;8. https://doi.org/10.7554/eLife.47999 PMID: 31219034

60. Kneuss E, Munafò M, Eastwood EL, Deumer U-S, Preall JB, Hannon GJ, et al. Specialization of the Drosophila nuclear export family protein Nxf3 for piRNA precursor export. Genes Dev. 2019; 33: 12081220. https://doi.org/10.1101/gad.328690.119 PMID: 31416967

61. Murano K, Iwasaki YW, Ishizu H, Mashiko A, Shibuya A, Kondo S, et al. Nuclear RNA export factor variant initiates piRNA-guided co-transcriptional silencing. EMBO J. 2019; 38: e102870. https://doi.org/10. 15252/embj.2019102870 PMID: 31368590

62. Ohtani H, Iwasaki YW, Shibuya A, Siomi H, Siomi MC, Saito K. DmGTSF1 is necessary for Piwi-piRISC-mediated transcriptional transposon silencing in the Drosophila ovary. Genes Dev. 2013; 27: 1656-1661. https://doi.org/10.1101/gad.221515.113 PMID: 23913921

63. Sienski G, Batki J, Senti K-A, Dönertas D, Tirian L, Meixner K, et al. Silencio/CG9754 connects the Piwi-piRNA complex to the cellular heterochromatin machinery. Genes Dev. 2015; 29: 2258-2271. https://doi.org/10.1101/gad.271908.115 PMID: 26494711

64. Yu Y, Gu J, Jin Y, Luo Y, Preall JB, Ma J, et al. Panoramix enforces piRNA-dependent cotranscriptional silencing. Science. 2015; 350: 339-342. https://doi.org/10.1126/science.aab0700 PMID: 26472911

65. Zhao K, Cheng S, Miao N, Xu P, Lu X, Zhang Y, et al. A Pandas complex adapted for piRNA-guided transcriptional silencing and heterochromatin formation. Nat Cell Biol. 2019; 21: 1261-1272. https://doi. org/10.1038/s41556-019-0396-0 PMID: 31570835

66. Quénerch'du E, Anand A, Kai T. The piRNA pathway is developmentally regulated during spermatogenesis in Drosophila. RNA. 2016; 22: 1044-1054. https://doi.org/10.1261/rna.055996.116 PMID: 27208314

67. Kotov AA, Adashev VE, Godneeva BK, Ninova M, Shatskikh AS, Bazylev SS, et al. piRNA silencing contributes to interspecies hybrid sterility and reproductive isolation in Drosophila melanogaster. Nucleic Acids Research. 2019; 47: 4255-4271. https://doi.org/10.1093/nar/gkz130 PMID: 30788506

68. Schindelin J, Arganda-Carreras I, Frise E, Kaynig V, Longair M, Pietzsch T, et al. Fiji: an open-source platform for biological-image analysis. Nat Methods. 2012; 9: 676-682. https://doi.org/10.1038/nmeth. 2019 PMID: 22743772

69. Oliphant TE. Guide to NumPy. Austin, Tex.: Continuum Press; 2015.

70. McKinney W. Data Structures for Statistical Computing in Python. Austin, Texas; 2010. pp. 56-61. https://doi.org/10.25080/Majora-92bf1922-00a

71. VanderPlas J, Granger B, Heer J, Moritz D, Wongsuphasawat K, Satyanarayan A, et al. Altair: Interactive Statistical Visualizations for Python. JOSS. 2018; 3: 1057. https://doi.org/10.21105/joss. 01057

72. Kent WJ, Sugnet CW, Furey TS, Roskin KM, Pringle TH, Zahler AM, et al. The human genome browser at UCSC. Genome Res. 2002; 12: 996-1006. https://doi.org/10.1101/gr.229102 PMID: 12045153

73. Robinson JT, Thorvaldsdóttir H, Winckler W, Guttman M, Lander ES, Getz G, et al. Integrative genomics viewer. Nat Biotechnol. 2011; 29: 24-26. https://doi.org/10.1038/nbt.1754 PMID: 21221095

74. Thorvaldsdóttir H, Robinson JT, Mesirov JP. Integrative Genomics Viewer (IGV): high-performance genomics data visualization and exploration. Brief Bioinformatics. 2013; 14: 178-192. https://doi.org/ 10.1093/bib/bbs017 PMID: 22517427 